OPEN ACCESS

Edited by:

James Chow,

University of Toronto, Canada

Reviewed by:

Ulrich W. Langner,

Lifespan, United States

Katsutoshi Mizuno,

Railway Technical Research Institute

(RTRI), Japan

Luca Bottura,

European Organization for Nuclear

Research (CERN), Switzerland

*Correspondence:

Mike D. Sumption sumption.3@osu.edu

Specialty section:

This article was submitted to

Radiation Oncology,

a section of the journal

Frontiers in Oncology

Received: 06 October 2021 Accepted: 13 December 2021 Published: 15 February 2022

Citation:

Collings EW, LU L,

Gupta N and Sumption MD (2022) Accelerators, Gantries,

Magnets and Imaging Systems for

Particle Beam Therapy: Recent Status and Prospects for Improvement.

Front. Oncol. 11:737837. doi: 10.3389/fonc.2021.737837

\section{Accelerators, Gantries, Magnets and Imaging Systems for Particle Beam Therapy: Recent Status and Prospects for Improvement}

\author{
Edward W. Collings ${ }^{1}$, Lanchun Lu ${ }^{2}$, Nilendu Gupta ${ }^{2}$ and Mike D. Sumption ${ }^{1 *}$ \\ ${ }^{1}$ Department of Materials Science and Engineering, College of Engineering, The Ohio State University, Columbus, $\mathrm{OH}$, \\ United States, ${ }^{2}$ Department of Radiation Oncology, The James Cancer Hospital and Solove Research Institute, Wexner \\ Medical Center and College of Medicine at the Ohio State University, Columbus, OH, United States
}

The paper begins by emphasizing the clinical and commercial importance of proton or other charged particle such as carbon ion therapy, refers to the manufacturers of such systems of which more than 120 are installed or under construction worldwide by April 2021. A general review of charged particle therapy systems refers to six manufacturers and provides in tabular form some details of systems installed in the US, Europe, Asia, and elsewhere. In a description of the principles of particle beam therapy a comparison is made of the properties of photons ( $\mathrm{x}$-rays) versus protons and protons versus carbon ions. A brief discussion of accelerators in general is followed by descriptions of cyclotrons (including the isosynchronous cyclotron and the synchrocyclotron) and synchrotrons. An interesting case study describes the evolution of a normal-conducting 220 ton cyclotron into an iron-free synchrocyclotron weighing only 5 tons. The general principles of beam handling and gantry design are described. Subsequent sections describe gantry magnets in detail - normal conducting gantry magnets, superconducting gantry magnets for proton- and carbon therapy. Mention is made of a novel CERN-designed superconducting toroidal gantry for hadron therapy, GaToroid. This device, operating under steady state current and magnetic field, is able to deliver a beam at discrete angles over a range of treatment energies. Also considered are low temperature superconducting (LTS) and high temperature superconducting (HTS) magnet windings, and the choice of REBCO conductors for cryogen-free carbon-ion gantries. Finally, the paper mentions an important "Prospect for Improvement", viz: the introduction of MRI image guidance. A well-known property of the particle beam as it passes through tissue is its energy dependent absorption that rises to a pronounced peak (the Bragg peak) at the end of its range. In order to take advantage of this effect the exact targeting of the tumor and positioning of the patient should be guided by imaging visualization using $\mathrm{X}$-ray, CT, and hopefully advanced MRI. Unlike MRI-guided photon therapy the direct interaction of the magnetic field with the charged particle beam presents a huge challenge such that MRI image-guided proton/particle therapy has not yet been available in clinical practice. 
Modeling studies have been undertaken on the general topic of beam-line/magnetic field interaction using, for example, the software GEANT4 (GEometry And Tracking) a platform for simulating the passage of charged particles through matter using a Monte Carlo method.

Keywords: particle beam therapy, protons, carbon ions, particle acceleration, cyclotron, image-guided particle beams, synchrotron

\section{INTRODUCTION: CLINICAL STATUS, MARKET REPORT AND PREDICTIONS, FACILITIES}

\subsection{Clinical Status 2014-2030}

2014: During year 2014 more than 140 treatment rooms were serving 14,500 patients

2015: In year 2015 only $0.5 \%$ of radiation-needy patients were treated with proton therapy.

2019: By year 2019330 patient treatment rooms are expected to be available, but even then only $1 \%$ of radiation-needy patients will be able to receive particle therapy.

2030: By year 2030 it is expected that 1200 to 1800 treatment rooms will be open to patients worldwide. But even 1800 rooms will allow only $5 \%$ of radiation-needy patients to receive particle therapy.

\subsection{Market Report and Predictions}

2000: In year 2000 the proton therapy market was valued at US\$ 1 million

2015: During year 2015 the market reached US\$ 800 million $(1,2)$

2019: In year 2019 the market is expected to reach more than US\$ 1 billion

During 2000-2014 the average annual growth rate was almost $15 \%$ p.a.

During 2010-2014 the growth rate underwent acceleration to $22 \%$ p.a.

2030: By year 2030 the proton therapy world market is expected to be worth US\$3.5 to 6.6 billion in response to an annual growth rate of $11 \%$ to $16 \%$.

\subsection{Facilities}

In 2015 there was reported to be 15 manufacturers or developers of ion therapy equipment, and it was predicted that by 2019 the market would be dominated by 5 of those companies. Important manufacturers include: Ion Beam Applications (IBA), Varian, Sumitomo, Hitachi, Toshiba, Siemens, and Mitsubishi, the latter having installed at least 11 proton- or carbon-ion centers in Japan. Of the more than 60 proton therapy facilities worldwide 25 are located in the US. After a slow start the proton therapy market began to accelerate in response to: (i) a growing recognition of the effectiveness of particle therapy, (ii) the recent introduction of lower cost compact systems and singletreatment-room centers. CERN, with its experience in proton accelerator technology (e.g. the LHC) continues to make important contributions to ion therapy. Proton/carbon-ion centers in Japan, Europe-and-Elsewhere, and the U.S. are listed in Tables 1-3, respectively.

An increasing interest is also being shown in carbon/heavyion therapy, institutions offering which include: The National Institute for Radiological Sciences (Chiba), Hyogo Ion Beam Medical Center (Hyogo), Gunma University Heavy Ion Medical Center (Gunma), Saga Heavy Ion Medical Accelerator in Tosu (Saga), and Shanghai Proton and Heavy Ion Center (SPHIC, Siemens). In 2019 the program "Next Ion Medical Machine Study" (NIMMS) was established to support R\&D based on CERN accelerator technology relevant to heavy ion therapy. It is also interesting to note that in a partnership between IBA and Toshiba the latter will become the Japanese distributor of IBA's Proteus ONE compact single-room proton therapy facility, and IBA will become Toshiba's carbon-therapy agent outside Japan. Carbon-ion centers worldwide are listed in Table 4.

\section{PRINCIPLES OF PARTICLE BEAM THERAPY}

\subsection{Reviews of Charged Particle Therapy and Systems}

Review articles on proton therapy $(6,7)$ begin by citing the work of Robert Wilson of the Lawrence Berkeley Laboratory who in a paper published in 1946 (8) was the first to point out the importance of the proton Bragg peak for targeted radiation therapy. As protons pass through tissue at velocity $v$ they continuously lose kinetic energy by inelastic Coulomb scattering against atomic electrons. The rate of energy loss, being proportional to $1 / v^{2}$, rises sharply as the protons slow down, the end of their range, and form the Bragg peak. Protons also undergo repulsive non-elastic interactions with atomic nuclei and will be deflected from their original path. The product of such interactions may be secondary protons, heavier ions, neutrons, and gamma rays. These non-elastic proton-nucleus interactions, although less frequent than proton-electron ones have a stronger effect (9).

For a detailed description of the history of proton therapy systems reference (7) is recommended. That same article also gives the locations and provides technical details (as of 2010) of 6 commercial proton beam therapy systems (PTS), viz: The IBA Proteus ${ }^{\circledR} 235$ PTS, Sumitomo PTS, Varian PTS, Still River Systems Monarch 250 (Mevion Medical Systems) PTS, Hitachi PROBEAT PTS, and Mitsubishi PTS. The Hitachi and Mitsubishi systems are based on synchrotron acceleration and the others on cyclotrons. Mitsubishi is particularly active in Japan; a list of their 
TABLE 1 | Partial listing of particle therapy centers in europe and elsewhere.

\begin{tabular}{|c|c|c|c|}
\hline Country & City & Institution & First patient \\
\hline China & Zibo & Wanje Proton Therapy Center & 2004 \\
\hline China & Lanzhou & Lanzhou Heavy Ion Therapy Research Center, Institute of Modern Physics, CAS & 2006 \\
\hline China & Shanghai & Shanghai Proton and Heavy lon Center & - \\
\hline Taiwan & Taipei & Chang Gung Memorial Hospital (CGMH) & 2012 \\
\hline Czech Republic & Prague & Proton Therapy Center Czech & 2012 \\
\hline France & Nice & Centre Laccassagne & 1991 \\
\hline France & Caen & Centre National de Radiotherapy & - \\
\hline France & Orsay & Centre Protontherapy de I'Institut Curie & - \\
\hline Germany & Berlin & $\mathrm{HMl}$ & 1998 \\
\hline Germany & Heidelberg & Heidelberg Ion Therapy Center & 2009 \\
\hline Germany & Munich & Rinecker & 2009 \\
\hline Germany & Dresden & Universitätsklinikum Carl Gustav Carus & 2014 \\
\hline Germany & Essen & Westdeutsches Protonentherapiezentrum Essen & 2013 \\
\hline Germany & Kiel & University Schleswig-Holstein (UC S-H) & na \\
\hline Germany & Marburg & Rhön-Klinikum & na \\
\hline Italy & Pavia & CNAO Pavia & 2009 \\
\hline Italy & Trento & Agenzia Provinciale Per la Protonterapia (AtreP) & 2012 \\
\hline Italy & Catania & Laboratori Nazionali del Sud & \\
\hline Korea & Seoul & Samsung Hospital & 2014 \\
\hline Korea & Ilsan & Korean National Cancer Center & 2007 \\
\hline Netherlands & Groningen & University Medical Center Groningen (UMCG) & - \\
\hline Poland & Krakow & Instytut Fizyki Jadrowej, Polish Acad. Sci. & 2013 \\
\hline Russia & Dimitrovgrad & Federal High-Tech Medical Center & 2013 \\
\hline Russia & St Petersburg & Center of Nuclear Medicine & 2016 \\
\hline Russia & Moscow & Institute for Theoretical and Experimental Physics & - \\
\hline Russia & Dubna & Joint Institute for Nuclear Research & - \\
\hline Sweden & Uppsala & Skandion Kliniken & 2013 \\
\hline Switzerland & Villigen & Paul Scherrer Institut & 1984 \\
\hline Saudi Arabia & Riyadth & King Fahd Medical City & 2015 \\
\hline South Africa & Somerset West & iThemba Labs & 1993 \\
\hline United Kingdom & Newport & The Rutherford Cancer Center South Wales & - \\
\hline United Kingdom & Clatterbridge & The Clatterbridge Cancer Center & - \\
\hline
\end{tabular}

installed proton- and carbon-ion systems is given in Table $\mathbf{5}$. A complete list of Japanese installations is given in Table $\mathbf{2}$ and lists of European and US particle therapy installations are given in

Tables 1, 3.

Excellent companions to the present document are two recently published reviews. In a paper entitled "Superconducting Magnets for Medical Accelerators" (11) S. Prestemon offers an introduction to hadron therapy and considers the challenges presented by superconducting technology. Cyclotrons and synchrotrons are reviewed, also gantries in general including TULIP (TUrning Linac for Proton Therapy) and ULICE (for Union of Light Ion Centres in Europe) which involves 20 European installations coordinated by CNAO (Pavia, Italy, see Section 4.1). L. Rossi assembled a document entitled "HITRI+ and

TABLE 2 | Particle therapy centers in Japan.

\begin{tabular}{|c|c|c|}
\hline Facility & Location & Ion Species \\
\hline Aizawa Proton Therapy Center (PTC) & Nagano Prefecture, Matsumoto & Proton \\
\hline Fukui Prefectural Hospital Proton Therapy Center & Yotsui, Fukui City & Proton \\
\hline Gunma University Heavy Ion Medical Center & Maebashi, Gunma & Carbon \\
\hline Hokaido University Hospital & Sapporo-shi, Hokaido & Proton \\
\hline Hyogo Ion Beam Medical Center & Tatsuno-shi, Hyogo & $\begin{array}{l}\text { Proton } \\
\text { Carbon }\end{array}$ \\
\hline Medipolis International Proton Beam Treatment Center & Ibusuki-shi, Kagoshima Prefecture & Proton \\
\hline National Cancer Center Hospital East & Kashiwa-shi, Chiba & Proton \\
\hline National Institute of Radiological Sciences & Inage-ku, Chiba-shi, Chiba & $\begin{array}{l}\text { Carbon } \\
\text { Heavy ion }\end{array}$ \\
\hline Nagoya Proton Therapy Center & Kita-ku, Nagoya & Proton \\
\hline Proton Medical Research Center, University of Tsukuba & Ibaraki Prefecture, Tsukuba & Proton \\
\hline Saga Heavy Ion Cancer Treatment Center & Tosu-shi, Saga Prefecture & Carbon \\
\hline Shizuoka Cancer Center & Sunto-gun, Shizuoka Prefecture & Proton \\
\hline Southern Tohoku PTC & Koriyama, Fukushima & Proton \\
\hline Tsuyama Chuo Hospital Proton Beam Cancer Center & Okayama & Proton \\
\hline Heavy Ion Medical Accelerator in Chiba, (HIMAC) & Chiba & $\mathrm{He}, \mathrm{Ne}, \mathrm{C}, \mathrm{Si}, \mathrm{Ar}$ \\
\hline
\end{tabular}


TABLE 3 | Proton therapy centers in the US $(3,4)$.

\begin{tabular}{|c|c|}
\hline Arizona & Mayo Clinic Proton Beam Therapy Program, Phoenix, AZ \\
\hline California & Scripps Proton Therapy Center, San Diego, CA \\
\hline California & James M. Slater, M.D. Proton Treatment and Research Center at Loma Linda \\
\hline University & Medical Center, Loma Linda, CA \\
\hline California & UCSF Ocular Tumor Proton Radiation Program, Crocker Nuclear Laboratory, San Francisco, CA \\
\hline California & UC Davis Cancer Center, Lawrence Livermore Nat'I Laboratory \& Tomotherapy Inc, CA \\
\hline Florida & Ackerman Cancer Center, Jacksonville, FL \\
\hline Florida & University of Florida Health Proton Therapy Institute, Gainesville, FL \\
\hline Florida & UF Health Cancer Center at Orlando Health, Orlando, FL \\
\hline Florida & Baptist Health South Florida, FL \\
\hline Illinois & Northwestern Medicine Chicago Proton Center, Chicago, IL \\
\hline Louisiana & Willis-Knighton Health System, Shreveport, LA \\
\hline Maryland & Maryland Proton Treatment Center, Baltimore, MD \\
\hline Massach'tts & Francis H. Burr Proton Center at Mass. General Hospital, Boston, MA \\
\hline Michigan & Beaumont Proton Therapy Center, Beaumont Hospital, Royal Oak, Ml \\
\hline Minnesota & Mayo Clinic Proton Beam Therapy Program, Rochester, Ml \\
\hline Missouri & Barnes Jewish Hospital (Washington University) St. Louis, MO \\
\hline Missouri & S. Lee Kling Proton Therapy Center at the Siteman Cancer Center, St Louis, MO \\
\hline New Jersey & ProCure Proton Therapy Center in partnership with Princeton Radiation Oncology Group and CentraState Healthcare System, Somerset, NJ \\
\hline New Jersey & Laurie Proton Therapy Center at Robert Wood Johnson University Hospital, New Brunswick, NJ \\
\hline Ohio & Cincinnati Children's/UC Health Proton Therapy Center, Liberty Township, $\mathrm{OH}$ \\
\hline Ohio & University Hospital's Seidman Cancer Center, Case Medical Center, Cleveland, $\mathrm{OH}$ \\
\hline Oklahoma & ProCure Proton Therapy Center, at the INTEGRIS Cancer Campus, Oklahoma City, OK \\
\hline Oklahoma & Stevenson Cancer Center, Oklahoma City, OK \\
\hline Pennsylvania & The Roberts Proton Therapy Center at University of Pennsylvania Health System,Philadelphia, PA \\
\hline Tennessee & Provision CARES Proton Therapy Center, Knoxville, TN \\
\hline Tennessee & St Jude Red Frog Events Proton Therapy Center, Memphis, TN \\
\hline Texas & Texas Center for Proton Therapy, Irving, TX \\
\hline Texas & M.D. Anderson Cancer Center's Proton Center, Houston, TX \\
\hline Virginia & Hampton University Proton Therapy Institute, Hampton, VA \\
\hline Washington & SCCA Proton Therapy Center, Seattle, WA \\
\hline Washington DC & Medstar Georgetown University Hospital, Washington DC \\
\hline
\end{tabular}

I-FAST: Next Eu programs for SC heavy ion therapy machine" (12) which outlined the status of ion therapy in Europe and Asia. Considered were HIT (the Heidelberg Ion Beam Therapy Centre, see Section 5.5), and HIMAC (the Heavy Ion Medical Accelerator in Chiba, see Section 5.7.1). Also described was the carbon ion superconducting gantry collaboration involving CNAO (Pavia, Italy, see Section 4.1), MedAustron (Weiner, Austria, see Section 4.1), CERN, and INFN.

\subsection{Radiation (Photons) versus lons}

In convention radiation (photon) therapy the absorption of $\mathrm{x}$ rays or gamma rays is intensive at the surface and decreases with distance into the subject. Thus in traditional therapy a high intensity of photons must be administered using the isocentric convergence technique to allow multiple beams with the diminished intensity focus at the tumor site. As a result, much of the photon's energy causes damage to healthy tissue.

TABLE 4 | Carbon-lon centers worldwide ${ }^{(a)(b)}$.

\begin{tabular}{|c|c|c|c|c|}
\hline Center Name & Institution & Country & City & $\begin{array}{l}\text { Date of } \\
\text { Operation }\end{array}$ \\
\hline The Center for lon Therapy and Research & MedAustron & Austria & $\begin{array}{l}\text { Wiener } \\
\text { Neustadt }\end{array}$ & 2017 \\
\hline Heavy Ion Research Facility & Institute of Modern Physics, Chinese Academy of Science & China & Lanzhou & 2006 \\
\hline Shanghai Proton and Heavy lon Center & Fudan University Shanghai Cancer Center & China & Shanghai & 2014 \\
\hline Heidelberg lon-Beam Therapy Center & University of Heidelberg & Germany & Heidelberg & 2009 \\
\hline Marburger lonenstrahl-Therapiezentrum & $\begin{array}{l}\text { Heidelburg University Hospital and University Hospital Giessen and } \\
\text { Marburg }\end{array}$ & Germany & Marburg & 2015 \\
\hline Foundation CNAO & National Centre for Oncological Treatment CNAO & Italy & Pavia (Milan) & 2011 \\
\hline Heavy Ion Medical Accelerator in Chiba & Japanese National Institute of Radiological Sciences & Japan & Chiba & 1994 \\
\hline Gunma University Heavy Ion Medical Center & Gunma University Heavy Ion & Japan & Gunma & 2012 \\
\hline Hyogo Ion Beam Medical Center & Medical Excellence JAPAN & Japan & Hyogo & 2001 \\
\hline $\begin{array}{l}\text { Kyusho International Heavy Particle Line Cancer } \\
\text { Treatment Center }\end{array}$ & & Japan & Tosu & 2013 \\
\hline i-Rock ion-beam Radiation Oncology Center & Kanagawa Cancer Center & Japan & Yokohama & 2015 \\
\hline
\end{tabular}

(a) Mostly based in a list published by the Chordoma Foundation.

(b) See also (5). 
TABLE 5 | Mitsubishi particle therapy systems (10).

\begin{tabular}{|c|c|c|c|}
\hline Facility & Location & Install, $\mathrm{n}$ year & Ion Species \\
\hline National Inst. Radiological. Sciences & Chiba-shi, Chiba & 1994 & Heavy lon \\
\hline Hyogo Ion Beam Medical Center & Tsuno-shi, Hyogo & 2001 & Proton/Carbon \\
\hline Shizuoka Cancer Center & Nagaizumi-cho, Shizuoka & 2003 & Proton \\
\hline Southern Tohoku Proton Therapy Center & Koriyama-shi, Fukushima & 2008 & Proton \\
\hline Fukui Prefectural Hospital Proton Therapy Center & Fukui-shi, Fukui & 2011 & Proton \\
\hline Gunma University Heavy Ion Medical Center & Maebashi-shi, Gunma & 2010 & Heavy lon \\
\hline Mediopolis Proton Therapy \& Research Center & Ibusuki-shi, Kagoshima & 2011 & Proton \\
\hline Saga Heavy Ion Medical Accelerator in Tosu & Tosu-shi, Saga & 2013 & Carbon \\
\hline Okayama University/Tsuyama Chuo Hospital Proton Beam Cancer Center & Tsuyama-shi, Okayama & 2016 & Proton \\
\hline Hakuhokai Group Osaka Proton Therapy Clinic & Osaka-shi, Osaka & $\begin{array}{l}\text { Under } \\
\text { Const'n }\end{array}$ & Proton \\
\hline Hyogo Prefecture Kobe Proton Therapy Center & Kobe-shi, Hyogo & $\begin{array}{l}\text { Under } \\
\text { Const'n }\end{array}$ & Proton \\
\hline
\end{tabular}

In contrast to photons, charged particles are "silver bullets" whose interactions with matter are characterized by the Bragg curve. The energy loss by charged particle through matter is described by this curve which rises to a maximum (the Bragg peak) just before the end of the particle's track. The peak occurs because the cross section for particle-matter interaction increases just before the particle comes to rest. In particle therapy the beam energy is adjusted either electrically (synchrotron accelerators) or by filters (cyclotron accelerators) to ensure that the Bragg peak occurs at the tumor site. Figures 1, 2 illustrate relative dose versus depth from the body surface for photon- and chargedparticle radiation. This indicates that while photon therapy is characterized by relatively high entrance and exit doses, proton therapy has not only a lower entrance dose but a negligible exit dose. It also shows that the position of the Bragg peak can be adjusted to ensure that the tumor receives the intended radiation. This allows patients to receive high radiation doses with low risk of collateral tissue damage.

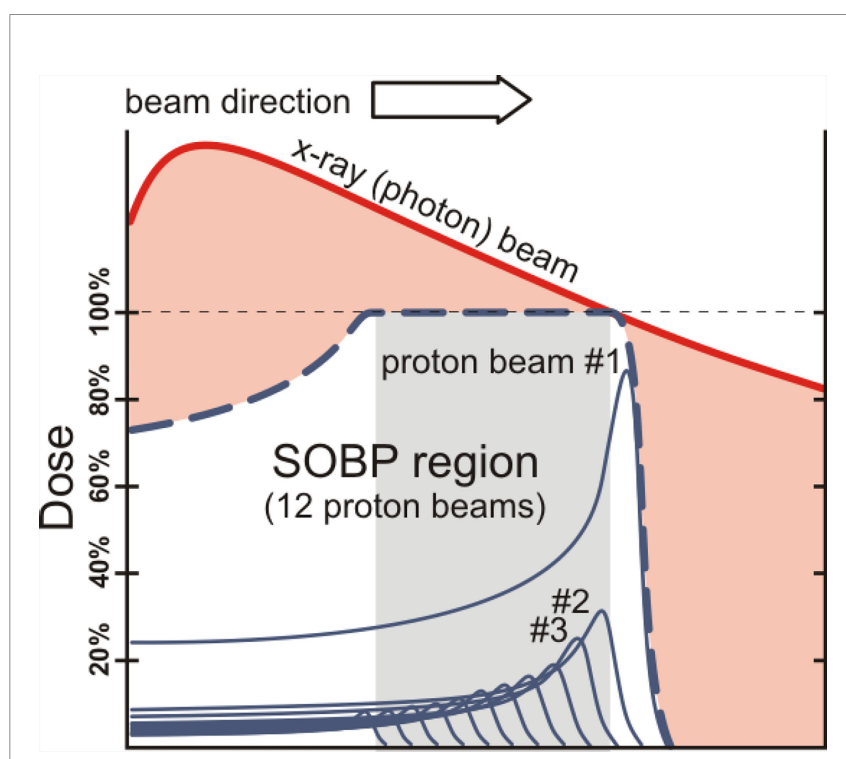

FIGURE 1 | Photons versus protons showing entrance and exit doses and the spread-out Bragg peak (SOBP) - after W.P. Levin et al. (13).

\subsection{Protons versus Carbon Ions}

The radiobiological effectiveness (RBE) of photon (traditional, $\mathrm{x}$ ray) therapy is arguably equivalent to that of proton therapy, however as illustrated in Figures $\mathbf{1}$ and 2, a relatively large proportion of the photon's energy is deposited in the entrance and exit healthy tissue regions. A distinct advantage of proton beams is that by modulating the energy to create a spread-out Bragg peak (SOBP) a large fraction of the beam energy can be deposited in the tumor site. This advantage is shared by heavy (in particular carbon) ions but in addition, being heavier than protons, they provide a higher RBE, that also increases with depth and rises to a maximum at the end of their range in the tumor region $(15,16)$.

Photon and proton radiation tend to produce only singlestrand DNA breakage. Since cells can repair such breaks damage to both strands is required for successful treatment. Carbon ions are able to produce double-strand breaks that cannot be repaired. In other words the RBE of carbon ions against DNA is up to

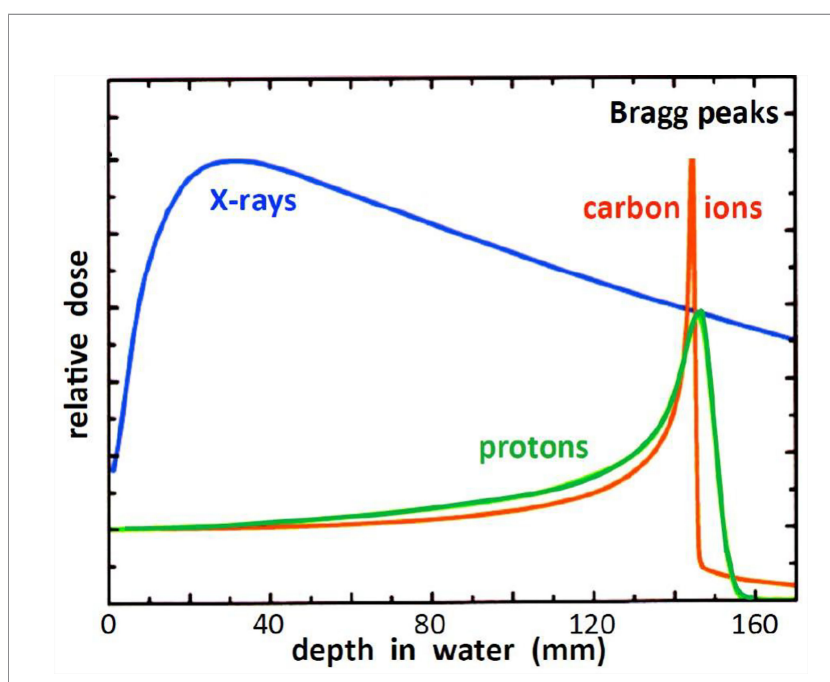

FIGURE 2 | Relative dose versus depth from the body surface for photonand charged-particle radiation - after (14). 
three times greater than that of $\mathrm{x}$-rays while protons are only slightly more effective. Whereas a full treatment with protons may require 30 sessions just four days may be needed for carbon.

For hadron therapy in general, ions of $\mathrm{He}, \mathrm{Li}, \mathrm{B}, \mathrm{N}, \mathrm{O}, \mathrm{Ne}(7)$, Ar, and Si (17) have also been considered, Section 4.2.

\section{BEAM HANDLING}

\subsection{Proton Acceleration and Handling}

Beams of ionized particles are accelerated and bent by the magnetic fields of cyclotrons, synchrotrons, and dipoles. If the particle velocity, $v$, is greater than one-third $c$, the velocity of light, a relativistic correction $\gamma=1 / \sqrt{ }\left[1-(v / c)^{2}\right]$ is applied to some of the equations. Thus the velocity $(\mathrm{m} / \mathrm{s})$ of an $E \mathrm{MeV}$ particle of rest mass $m_{0}$ can be deduced from $E=m_{0} c^{2}(\gamma-1)$ in SI units (1 $\left.\mathrm{MeV}=1.602 \times 10^{-13} \mathrm{~J}\right)$. The bend radius $\rho$ of a beam of particles of momentum $p=m_{0} \gamma$ and charge $q$ deflected by a magnetic field $B$ is given in SI units by:

$$
\rho=(p / q) / B=\left(m_{0} \gamma / q\right) /(v / B)
$$

The quantity $p / q=\left(m_{0} \gamma / q\right)$ which has the units Tm is known as the "magnetic rigidity", e.g (5). It represents the reluctance of the ion beam to being deflected by the field $B$. The energydependent rigidities of some proton and carbon beams are listed in Table 6, see also Appendix A. The table demonstrates that heavy ions beams are more difficult to deflect than proton beams and hence require stronger magnetic fields

\subsection{Heavy lon (Hadron) Acceleration and Handling}

Hadrons are accelerated in circular paths by cyclotrons or synchrotrons. In a modification of Equation (1) the path radius is proportional to $(M / Q)(v / B)$ in which $M$ represents ionic mass (number of protons and neutrons in the nucleus) and $Q$ represents the ionic charge (number of stripped electrons). Thus as suggested in (7) a hadron synchrotron can accelerate a long list of $0.5-(Q / M)$ ions, in particular:

hydrogen (accelerated as $\mathrm{H}_{2}^{+1}$ molecule). $\mathrm{Q} / M=0.5$ helium, $Q / M=2 / 4.0=0.5$

lithium, $Q / M=3 / 6.9=0.43$

boron, $Q / M=5 / 10.8=0.46$

carbon, $Q / M=6 / 12.0=0.50$

nitrogen, $Q / M=7 / 14.0=0.50$

oxygen, $Q / M=8 / 16.0=0.50$

neon, $Q / M=10 / 20=0.50$

argon $Q / M=18 / 39.9=0.45$

silicon $\mathrm{Q} / \mathrm{M}=14 / 28.1=0.50$

Protons are the commonly used ions, accelerated to 220-230 $\mathrm{MeV} / \mathrm{u}$ they can travel $30-33 \mathrm{~cm}$ into the body. Fewer systems use carbon ions which have about the same range at $400 \mathrm{MeV} / \mathrm{u}$.

\section{PARTICLE ACCELERATION}

\subsection{Accelerators and Systems in General}

For injection into a synchrotron that accelerates them to final beam energy (21) particles are typically pre-accelerated by a radiofrequency quadrupole (RFQ) cavity accelerator $(7,22)$ followed by a drift-tube linear accelerator (DTL) $(7,23)$. The numerous processes that exist for injection into cyclotrons have been discussed in detail by Mandrillon (24). Some commercial accelerators are listed in Table 7.

CERN, with its experience in proton accelerator technology (e.g. the LHC) continues to make important contributions to ion therapy. Some 20 years ago CERN initiated the program "Proton Ion Medical Machine Study" (PIMMS) whose purpose was to produce a synchrotron tailored to treat tumors with protons and carbon ions. The design evolved into a proton and carbon ion machine built for CNAO (Italy's National Center for Oncological Hadron Therapy, Pavia Italy, Tables 1 and 4). Subsequently MedAustron (Weiner, Austria, Tables 1 and 4) with technical support from CERN based its clinic on the CNAO design. In 2019 the program "Next Ion Medical Machine Study" (NIMMS, led by M. Vretenar) was established to support R\&D based on CERN accelerator technology relevant to heavy ion therapy.

TABLE 6 | lon energies and dose penetration depths, also calculated magnetic rigidities, fields for 1.5-m-Bend radius, and 2-T-Dipole bend radii.

\begin{tabular}{|c|c|c|c|c|c|c|c|}
\hline $\begin{array}{l}\text { Ion } \\
\text { Species }\end{array}$ & $\begin{array}{c}\text { Beam Energy, } E, \\
M e V / u\end{array}$ & $\begin{array}{l}\text { Beam Energy, } E, \\
\text { GeV }\end{array}$ & $\begin{array}{c}\text { Dose Depth, } \\
\text { cm }\end{array}$ & Reference & $\begin{array}{c}\text { Magn.Rigidity, } R, \\
\mathrm{Tm}\end{array}$ & $\begin{array}{l}\text { Field for1.5 mBend Radius, } \\
\qquad B, T\end{array}$ & $\begin{array}{c}2 \text { T BendRadius, } \rho, \\
\mathrm{m}\end{array}$ \\
\hline Proton & 70 & & & (5) p.2751 & 1.231 & 0.8 & \\
\hline Proton & 175 & & 20 & (18) p.244 & 2.001 & 1.3 & \\
\hline Proton & 220 & & 30 & (19) p.1 & 2.268 & 1.5 & 1.13 \\
\hline Proton & 230 & & 33 & (20) p.3 & 2.324 & 1.5 & 1.16 \\
\hline Proton & 250 & & - & $\begin{array}{l}\text { (5) p.2751 } \\
\text { (20) p.16 }\end{array}$ & 2.43 & 1.6 & 1.22 \\
\hline Carbon & 425 & 5.10 & - & (20) p.16 & 6.582 & 4.4 & 3.29 \\
\hline Carbon & 430 & 5.16 & 30 & (19) p.1 & 6.627 & 4.4 & 3.31 \\
\hline
\end{tabular}


TABLE 7 | Accelerators for proton and/or carbon-lon therapy - some commercial vendors.

\begin{tabular}{|c|c|c|}
\hline Accelerators* & Vendor & Notes \\
\hline Cyclotrons & Varian/ACCEL Instruments & $250 \mathrm{MeV}$ superconducting isochronous cyclotron - see also (7) \\
\hline “ & $\mathrm{IBA}$ & $\begin{array}{l}\text { Proteus }{ }^{\circledR} 235 \text {, uses } 230 \mathrm{MeV} \text { normal-conducting isochronous cyclotron; subsequently Proteus ONE }{ }^{\circledR} \text { system uses } \\
230 \mathrm{MeV} \text { superconducting synchrocyclotron, S2C2 }\end{array}$ \\
\hline " & ProNova & ProNova SC360 superconducting isochronous cyclotron \\
\hline " & $\begin{array}{l}\text { Mevion Medical Systems (Still } \\
\text { River Systems, Inc) }\end{array}$ & $\begin{array}{l}\text { Mevion S250, } 250 \mathrm{MeV} \text { superconducting synchrocyclotron with gantry-mounted } 9 \mathrm{~T} \text { cryo-cooled } \mathrm{Nb}_{3} \mathrm{Sn} \text {-wound } \\
\text { coils }\end{array}$ \\
\hline " & Sumitomo & Normal-conducting $230 \mathrm{MeV}$ isosynchronous cyclotron; cf. IBA's first machine \\
\hline Synchrotrons & Hitachi & PROBEAT-V Slow-cycling 70-250 MeV synchrotron \\
\hline “ & Mitsubishi & $\begin{array}{l}70-250 \mathrm{MeV} \text { synchrotrons for protons or carbon ions, } \\
9 \text { installed, others under construction }\end{array}$ \\
\hline " & Optivus Technology & Synchrotron, 8 m diameter, continuously variable $70-250 \mathrm{MeV}$ protons, cf. Loma Linda PBTS \\
\hline " & Siemens & Synchrotron, 20 m diameter, 50-250 MeV/u (protons) and 85-430 MeV/u (carbon ions) \\
\hline " & Toshiba & Synchrotron, 10 m diameter, $70-235 \mathrm{MeV}$ \\
\hline
\end{tabular}

${ }^{*}$ Cyclotrons vs synchrotrons: The latter do not require energy degraders since the ions beam is accelerated to the desired energy.

\subsection{Synchrotrons}

Therapy synchrotrons yield beams of relatively low intensity and of variable energy. Protons are injected into a ring of dipoles at 2$7 \mathrm{MeV}$ and accelerated up to $70-250 \mathrm{MeV}$ as the dipole field is ramped. Synchrotrons can be up to $20 \mathrm{~m}$ in diameter. The first hospital-installed proton therapy system was based on a Fermilab synchrotron to be further developed by Optivus Technology. Other vendors such as Hitachi, Mitsubishi, Toshiba, and Siemens followed suit, Table 7. But as Jongen has pointed out (7) about $75 \%$ of today's proton therapy systems are based on cyclotron technology

\subsection{Cyclotrons}

The cyclotron consist of an RF system situated between the poles of a normal-wound or superconducting electromagnet. Considered below are the classical "traditional" cyclotron, the isochronous cyclotron, and the synchrocyclotron.

\subsubsection{The Conventional Cyclotron}

This cyclotron incorporates a fixed-field electromagnet. The RF system consists of a pair of hollow D-shaped drift cavities excited by an oscillator of fixed frequency, $f$, given by $2 \pi f=(q / m) B$ where $q$ and $m$ are the charge and mass of an assumed non-relativistic particle and $B$ is the field strength. Early on a SumitomoProNova collaboration produced a $230 \mathrm{MeV}$ conventional normal-conducting cyclotron. In 1989 Blosser et al. (25) reported on the development at Michigan State University (MSU) of a $100 \mathrm{MeV}$ superconducting cyclotron for installation at Detroit's Harper Hospital. The cyclotron and beam delivery system are gantry mounted and will rotate through a $360^{\circ}$ arc about the patient.

\subsubsection{The Isochronous Cyclotron}

In this cyclotron the RF frequency is fixed but $B$ varies with radius. Furthermore an azimuthal variation in $B$ provides a strong focussing effect and constrains the particles in their spiral paths. The isochronous cyclotron also called the azimuthal varying field (AVF) cyclotron, is used in many of today's systems: (i) IBA offered a resistive magnet Proteus ${ }^{\circledR} 235$ system which in 2010 was operating in 9 locations (7); (ii) ProNova produced a $230 \mathrm{MeV}$ superconducting isochronous cyclotron which was about $1 / 2$ the diameter and $1 / 4$ the weight of its resistive one mentioned above; (iii) Varian/ACCEL's ProBeam system incorporates a $250 \mathrm{MeV}$ isochronous cyclotron, the interesting feature of which is its use of four drift cavities instead of the usual two.

\subsubsection{The Synchrocyclotron}

In the synchrocyclotron the RF frequency, $f_{R}$, is decreased continuously in synchronism with the increasing velocity, $v$, of the particle within the relativistic regime. Thus $f_{R}=(q / m \gamma) B$, where $\gamma=1 / \sqrt{ }\left[1-(v / c)^{2}\right]$. The synchrocyclotron may deploy only one $\mathrm{D}$ whose potential oscillates with respect to ground. The circulating particles accelerate as they drift into and out of the D. The IBA Proteus ${ }^{\circledR} 235$ system, a successor to their ProteusOne ${ }^{\circledR}$, incorporates their model S2C2 superconducting ( $\mathrm{NbTi}$ ) synchrocyclotron and operates at a fixed $230 \mathrm{MeV}$ (26). In 1989 Blosser et al. (27) reported on the design of a $250 \mathrm{MeV}$ superconducting gantry-mounted synchrocyclotron system for proton therapy.

\subsection{Evolution of Ion-Beam Therapy Accelerators}

A Sumitomo-ProNova Solutions collaboration produced a normal-conducting $230 \mathrm{MeV}$ cyclotron $4.4 \mathrm{~m}$ in diameter weighing 220 tons. The smaller ProNova SC360 system used a superconducting $230 \mathrm{MeV}$ isochronous cyclotron $2.8 \mathrm{~m}$ in diameter weighing 50 tons. The Massachusetts Institute of Technology (MIT) in collaboration with ProNova has been working on the design and construction of an iron-free variable energy (70-230 MeV) synchrocyclotron also $2.8 \mathrm{~m}$ in diameter but weighing only 5 tons (28). Several advantages accrue from the use of this accelerator: (i) its variable-energy capability removes the need for graphite energy degradation, (ii) its low weight would make it very attractive for gantry mounting, (iii) gantry mounting eliminates the need for beamdirecting magnets. Taken together these advantages lead to an attractive proton beam therapy system. 


\section{COMPONENTS OF THE ION BEAM THERAPY SYSTEM}

\subsection{Beam Energy Adjustment}

Before it enters the treatment area or gantry the ion beam requires energy adjustment. Therapy cyclotrons generate a fixed-energy beam of typically $230 \mathrm{MeV}$, the needed energy variation between 60 and $230 \mathrm{MeV}$ being achieved by passing the beam through an "energy degrader, an absorber of variable thickness such as two opposite-facing wedges of graphite. These are followed by a magnetic analyzer consisting of a combination of dipole magnets and collimators (20). The "Energy Selection System" of Ion Beam Applications S.A. (IBA) allows tuning from $60-230 \mathrm{MeV}$ in less than 1 second. Although the beam loss by degradation can be as much as a factor of one hundred or more typical cyclotrons deliver sufficient beam intensity to make up for this. With regard to synchrotron sources, rapid energy variation can be achieved by extracting the ion beam at different times in the acceleration cycle (20). The synchrotron can deliver proton beams in more than 90 energy steps between $73 \mathrm{MeV}$ and $222 \mathrm{MeV}$ corresponding to penetration depths in water of 4 to $31 \mathrm{~cm} \mathrm{(29).}$

\subsection{The Gantry}

In the present context a gantry is a massive structure that rigidly holds in place the guidance magnets of ion beam therapy. A typical magnet arrangement is shown in Figure 3. The figure also indicates the so-called "isocenter" or axis about which the gantry rotates thereby enabling the ion beam to enter the tumor in all directions. The term "gantry" may refer to the mechanical structure, the magnet string (5), or the entire system as illustrated in Figure 4.

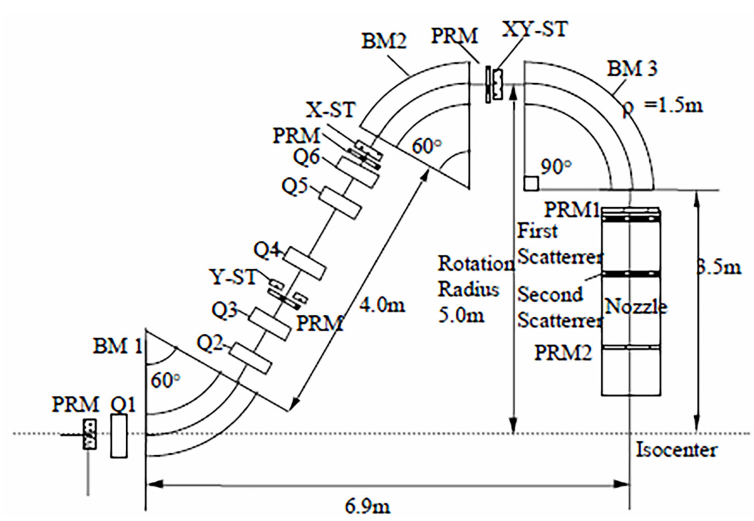

FIGURE 3 | Pavlovic (30) layout of magnets in an ion therapy gantry - after (5). In this example two bending dipoles (BM 1 and BM 2) offset the beam $5 \mathrm{~m}$ from the isocenter and dipole BM 3 bends the beam back towards the isocenter and the patient platform. In other systems BM 1 and BM 2 would have other but equal bend angles [e.g. $45^{\circ}(19)$ ], and still others may employ only two bending magnets (e.g. $\mathrm{BM} 1$ with $45^{\circ}$ or $60^{\circ}$ and $\mathrm{BM} 3$ with $135^{\circ}$ or $150^{\circ}$ ) to achieve the same result $(31,32)$.

\subsection{Components of the Magnet String and Gantry}

Figure 3 illustrates a Pavlovic-type (30) arrangement of magnets and devices in an ion therapy gantry. The beam is bent by three dipole magnets BM 1, BM 2, and BM 3, and kept in focus by six quadrupole magnets Q1 to Q6. The beam enters the gantry through a monitor PRM that monitors and records the center of the beam and its size. Three other PRMs are associated with steering magnets for beam trajectory correction and two more, PRM1 and PRM2, are located in the nozzle. Several nozzle arrangements have been used to passively or actively spread the $\mathrm{mm}$-size beam over a treatment area that may be as large as $30 \mathrm{~cm}^{2}$ (5, 34). Described in detail by (31) are: (i) uniform scanning nozzles, (ii) pencil scanning nozzles, (iii) single scattering nozzles, and (iv) double scattering nozzles. The latter version is represented in Figure 3.

\subsection{Bending Dipoles: Momentum Acceptance}

During a typical treatment session a proton beam energy may need to be varied from 70 to $250 \mathrm{MeV}$. This produces a change in rigidity of from 1.231 to $2.431 \mathrm{Tm}$ requiring the field of a $1-\mathrm{m}$ radius dipole to track the beam energy and hence increase from 1.23 to $2.43 \mathrm{~T}$. In the absence of field-change a typical normal dipole will accept a beam momentum change, $d p / p$, of less than $1 \%$ ( (35), p.2). Tumors are typically scanned in layers $5 \mathrm{~mm}$ deep enabled by a momentum sweep of $1 \%$ ( (35), p.4), the layerscan taking of order $100 \mathrm{~ms}$. A $d p / p$ of $1 \%$ corresponds to a beam energy change $d E / E=2 d p / p=2 \%$. Thus to control a $100 \mathrm{MeV}$ beam (proton magnetic rigidity $1.483 \mathrm{Tm}$ ) a 1 -m-radius dipole would require a field change of $15 \mathrm{mT}$. On this basis the average sweep rate of the scan is $150 \mathrm{mT} / \mathrm{s}$ (20 times faster than LHC). Taking another approach, a $d E / E$ of $2 \%$ implies 50 scanning steps covering the energy range from 70 to $250 \mathrm{MeV}$ and a 1-m-radius dipole field range of 1.23 to $2.43 \mathrm{~T}$. At $100 \mathrm{~ms} / \mathrm{step}$ this leads to

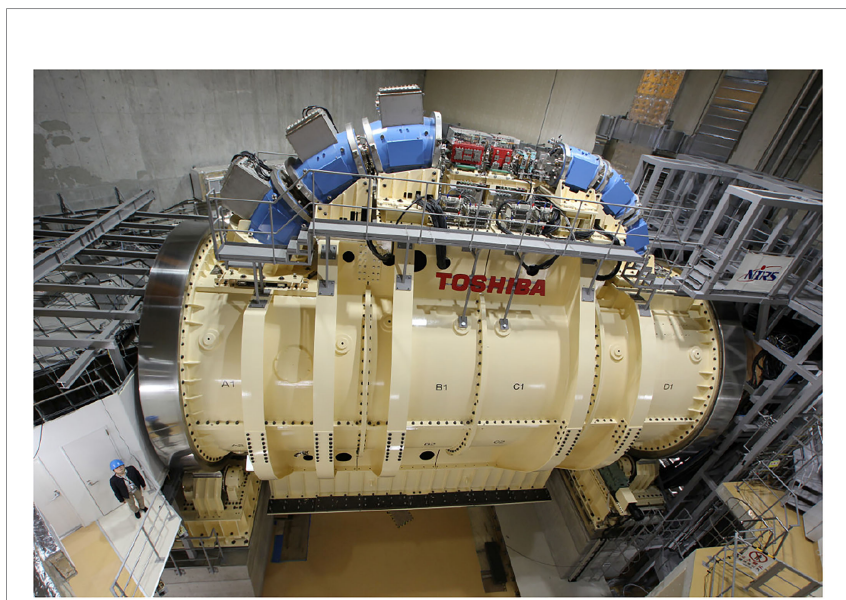

FIGURE 4 | Toshiba's gantry with superconducting technology capable of $360^{\circ}$ rotation about the isocenter - after (33). 
an average sweep rate of $240 \mathrm{mT} / \mathrm{s}$. From another standpoint, at $5 \mathrm{~mm}$ per layer a proton-beam-depth range of $10-35 \mathrm{~cm}$ would require 50 scanning steps, leading to the same result.

Sweep rates of hundreds of $\mathrm{mT} / \mathrm{s}$ cause stability and energyloss problems for superconducting magnets. So when considering a gantry upgrade from normal-conducting to superconducting magnets these high ramp rates created a demand for achromatic bending dipoles with wide momentum acceptances, such $d p / p=5-10 \%, 25 \%$, and 40-50\% [(35), p.3]. The use of such magnets, which enable the entire energy range to be covered in just a few steps, eases the ramp-rate requirement.

\subsection{Normal-Conducting Gantry Magnets}

As outlined in (20) the use of normal-conducting iron-core dipole magnets with their maximum bore field, $B$, of about 1.8 $\mathrm{T}$ governs the size of most commercial gantries. At a typical 250 $\mathrm{MeV}$ proton beam rigidity, $R=2.43 \mathrm{Tm}$ the $1.8 \mathrm{~T}$ field calls for a bending radius $\rho=R / B=1.35 \mathrm{~m}$. The addition of $3.5 \mathrm{~m}$ for distance from the nozzle entrance to the isocenter leads to a gantry rotation radius of about $5 \mathrm{~m}$ (Figure 3 ). Thus proton gantries are about 10-12 $\mathrm{m}$ in diameter, 7-10 $\mathrm{m}$ long (Figure 3 also (18)) and weight about 100-200 tons (20). Further details are provided in Appendix B.

Much larger than the above proton gantries is the famous carbon-ion gantry of the Heidelberg Ion Beam Therapy Centre (HIT). The maximum treatment beam energy of $425 \mathrm{MeV} / \mathrm{u}$ yields a beam rigidity of $6.58 \mathrm{Tm}$ (Table 6) which calls for a $1.8 \mathrm{~T}$ dipole radius of $\rho=R / B=3.65 \mathrm{~m}$. The supporting gantry is $\approx$ $12 \mathrm{~m}$ in diameter, $\approx 21 \mathrm{~m}$ long, and the overall system (including 135 tonnes of magnets) weighs $\approx 650$ tonnes (36). These statistics signaled the beginning and end of normal-conducting carbonion gantries and ushered in the need for superconducting magnets (36). In conclusion we note that HIT should not be confused with HITRI+ which stands for "Heavy Ion Therapy Research Initiative" a design study to assess the relative merits of CT and CCT magnets (Sections 5.7.2 and 5.7.4) for synchrotrons (Section 4.2) and CT/CCT and toroids (Section 5.7.6) for gantries.

\subsection{Superconducting Gantry Magnets for Proton Therapy}

\subsubsection{Conventionally Wound Magnets}

It is easy to argue that proton gantries are adequately served by normal-conducting magnets. Nevertheless superconductivity has enabled several improvements to gantry and magnet design. Alonso and Antaya (18) considered the size advantage accompanying the substitution of $2 \mathrm{~T}$ normal-conducting dipoles with superconducting ones with $4 \mathrm{~T}$ or higher fields. The smaller bend radius and smaller size were estimated to reduce the gantry diameter by about $3 \mathrm{~m}$. Alonso et al. have also described a novel gantry concept being developed at the time by ProNova Solutions based on achromatic (9\% momentum acceptance) combined-function magnets. In that arrangement a $60^{\circ}$ bend was followed by a set of magnets (possibly a pair) contributing to a final $150^{\circ}$ bend. The total mass of the magnets was estimated to be less than 5 tons. The "direct replacing" of normal-conducting magnets with superconducting ones should not only lower the weight but also the cost of the gantry. With these advantages in mind Bontoiu and Sanchez-Segovia (37) went on to model a lattice of 36 combined-function superconducting magnets. Inspired by LHC technology the combined-function magnet consists of one layer of quadrupole coils on top of one layer of $\cos -\theta$ dipole coils. Combinedfunction magnets possess much larger momentum acceptances than do individual dipoles and quadrupoles. In this case a fixedfield beam energy variation of $50 \mathrm{MeV}$ could be accommodated, enabling an energy range of 100 to $250 \mathrm{MeV}$ to be covered in only three steps. The superconducting gantry design study of Wan et al. (38) was motivated not only by size, weight, and cost considerations but also the need for large energy acceptance. Their design achieved an energy acceptance of $\pm 21 \%$ enabling beams of $150-30 \mathrm{MeV}$ to be bent without field ramping. Wan et al's compact fixed-field-alternating-gradient (FFAG) gantry, or magnet string, consisted of three groups of seven achromatic $3.2 \mathrm{~T}$ superconducting magnets, each group creating a $90^{\circ}$ bend (38).

\subsubsection{Canted Cosine Theta Magnets}

It was shown in 1970 that an overlaid pair of solenoidal coils tilted in opposite directions could generate a dipolar field normal to the solenoidal axis (39). Exploited in recent years by the superconducting magnet group at the Lawrence Berkeley National Laboratory (LBNL) this tilted-double-helix magnet is now referred to as canted cosine theta, CCT. By suitably modifying the winding scheme quadrupoles and higher order multipoles can also be generated (18). Not only that, but a combined-function magnet can be produced by winding a CCT dipole on top of a CCT quadrupole (40). In pursuing CCT technology the LBNL group went on to introduce a new magnet concept - the alternating-gradient canted cosine theta (AGCCT) magnet $(35,40)$. In this magnet the inner quadrupole winding consists of multiple sections with the current reversed between sections, e.g. 5 sections for a winding designated FDFDF. LBNL's design can be characterized as fixed-field alternatinggradient since its large momentum acceptance $(\sim 25 \%)$ enables a large energy range to be transmitted without changing the field. The advantages claimed for gantries incorporating the new magnet design are: (i) an order of magnitude reduction in weight, (ii) possible size reduction, e.g. diameter $5 \mathrm{~m}$ and length $8.3 \mathrm{~m}$, and (iii) rapid scanning (35). Gantry layouts described by the LBNL group are: (i) three groups of seven achromatic 3.2 $\mathrm{T}$ superconducting magnets (with sextupole and octupole components in the middle five of each group), with each group creating a $90^{\circ}$ bend (38), (ii) two $75^{\circ}$ AGCCT bending magnet groups to offset the beam $2.5 \mathrm{~m}$ "above" the exocenter followed by one $90^{\circ}$ AGCCT group (35), (iii) one normal-conducting 45 dipole to deflect the beam "upwards" followed by a single $135^{\circ}$ bend to guide the beam back to the exocenter (40). As with other superconducting gantry systems the magnets are intended to be conduction cooled. 


\subsubsection{Superconductors for Magnet Windings}

Several practice CCT-based coils were wound at LBNL using parallel stacks $(6 \times 1$ and $8 \times 1)$ of insulated square $(1.6 \mathrm{~mm} \mathrm{x}$ $1.6 \mathrm{~mm}$ ) NbTi wire. During the above studies the LBNL group conducted a detailed evaluation of the advantages and disadvantages of $\mathrm{Nb}_{3} \mathrm{Sn}, \mathrm{MgB}_{2}$, and HTS wires and selected NbTi for a number of reasons [(35) Table 8, see also (41)]. It is interesting to note that the Toshiba Corporation has been considering HTS magnets with the aim of reducing gantry size and has designed and fabricated a model magnet (42).

\subsubsection{The MEVION S250}

The MEVION S250 system is unconventional in that the gantry carries no beam-transport magnets. Instead the proton beam emanates directly from a gantry-mounted $250 \mathrm{MeV}$ superconducting synchrocyclotron whose magnet, wound with $\mathrm{Nb}_{3} \mathrm{Sn}$ wire, and cryocooled to $4 \mathrm{~K}$, delivers a central field of $9 \mathrm{~T}$. Weighing only 22 tons Mevion's SCS is much lighter than comparable machines, e.g. IBA's 50 ton $230 \mathrm{MeV}$ superconducting synchrocyclotron (see Figure 5). At least 7 S250 systems are presently operational and the S250i with pencil beam scanning is also available, Table 8.

\subsection{Superconducting Gantry Magnets for Hadron Therapy \\ 5.7.1 Background}

Freed from the need for an iron core, superconducting magnets can be much stronger in field and lighter than their normalconducting counterparts and hence are suitable as hadrontherapy gantry magnets. Whereas a $230 \mathrm{MeV}$ proton beam can achieve a dose depth of $33 \mathrm{~cm}$, carbon ions need to be accelerated to $400 \mathrm{MeV} / \mathrm{u}(4.8 \mathrm{GeV})$. Accordingly the magnetic rigidities $(M=\rho B)$ of these beams are 2.32 and 6.35 Tm, respectively. The dipole field needed to achieve a $1.5 \mathrm{~m} 230$ $\mathrm{MeV}$ proton-beam bend radius is $1.5 \mathrm{~T}$ whereas the $400 \mathrm{MeV} / \mathrm{u}$ carbon-ion beam requires $4.2 \mathrm{~T}$ (Table 6), achievable only in a superconducting magnet. Such magnets can provide very small carbon-beam bend radii; given that $\rho=6.35 / B$ radii of less than $1 \mathrm{~m}$ are possible.

The advantage of carbon ions is that, being heavier than protons, they provide a higher RBE. After their pioneering work with proton beams in $1954 \mathrm{LBNL}$ moved on to helium ions in 1957, and neon ions in 1975, but ended all radiotherapy programs in 1992 (15). In 1994 carbon ion radiotherapy (CIRT) was picked up by Japan's National Institute of Radiological Sciences (NIRS) using the Heavy Ion Medical Accelerator in Chiba (HIMAC). Other centers were soon to follow; five in Japan and others in Austria, China, Germany and Italy ( $(7,15)$ and Table 4). In spite of its innovative pioneering work the US does not house a single CIRT center, although initial planning has been announced for the establishment of such centers in California and Colorado $[(43,44)$ see also $(45)]$.

\subsubsection{Conventionally Wound LTS Magnets for Carbon Therapy}

In 2012 Iwata et al. and others from the Toshiba Corporation reported on the design of a rotating gantry system with superconducting magnets for carbon ion therapy (46). Then in 2013 Toshiba received an order from NIRS for such a system, the world's first (33). The cylindrical gantry is depicted in Figure 4. In addition to a pair of scanning magnets, three pairs of steering magnets and beam profile monitoring magnets it consisted of 10 superconducting combined-function bending magnets. One of the design goals was to achieve a compact superconducting gantry, much smaller than HIT's room-temperature one and comparable in size (length $13 \mathrm{~m}$, radius $5.5 \mathrm{~m}$ ) to existing proton gantries. The 10 bending magnets, BM1-10, of the Toshiba/NIRS $430 \mathrm{MeV} / \mathrm{u}$ magnet system were arranged in 3 groups - BM1-3, BM4-6 and BM7-10, Table 9.

The superconducting combined-function coils have a layered structure: the innermost 8 layers being $\cos (2 \theta)$ quadrupoles and the outer 26 layers being $\cos \theta$ dipoles. The small momentum acceptance of the system necessitated the use of low AC-loss NbTi wire - $10 \mu \mathrm{m}$ filament diameter with CuNi barriers (47). The magnets were conduction cooled with $1.5 \mathrm{~W} / 4.2 \mathrm{~K}$ GiffordMcMahon cryocoolers. Three sets were mounted on each of BM1-6 and four sets on each of BM7-10 for a total of 34 cryocoolers (47). An even more compact gantry was described by Iwata et al. (48). Consisting of only three identical $90^{\circ}$ combined-function magnets it was only $5.1 \mathrm{~m}$ in length and $2.5 \mathrm{~m}$ in radius. Designed for $430 \mathrm{MeV} / \mathrm{u}$ carbon ions and hence a beam rigidity of $6.627 \mathrm{Tm}$ each magnet has a bending radius $\rho=1.32 \mathrm{~m}$ and a maximum bore field $B=5.02 \mathrm{~T}$. As described and depicted in (48) the combined-function magnet consisted of a 20 -layer of $\cos \theta$ dipole winding on top of a 6 -layer $\cos (2 \theta)$ quadrupole winding.

TABLE 8 | MEVION S250 compact proton therapy systems.

\begin{tabular}{|c|c|c|}
\hline Facility & Location & Status \\
\hline MedStar Georgetown University Hospital & Washington, DC & Under installation \\
\hline Stevenson Cancer Center & Oklahoma City, OK & Clinically accepted \\
\hline UF Health Cancer Center at Orlando Health & Orlando, FL & Clinically operational \\
\hline Ackerman Cancer Center & Jacksonville, FL (a) & Clinically operational \\
\hline Seidman Cancer Center, University Hospitals & Cleveland, $\mathrm{OH}$ & Clinically operational \\
\hline Siteman Cancer Center, Barnes Jewish Hospital, Washington University & St Louis, Missouri & Clinically operational \\
\hline Robert Wood Johnson University Hospital & New Brunswick, NJ & Clinically operational \\
\hline ZON-PTC at Brightlands Maastricht Health & Maastricht, Netherlands (b) & \\
\hline
\end{tabular}

(a) S250 operational; S250i under contract, (b) S250i. 


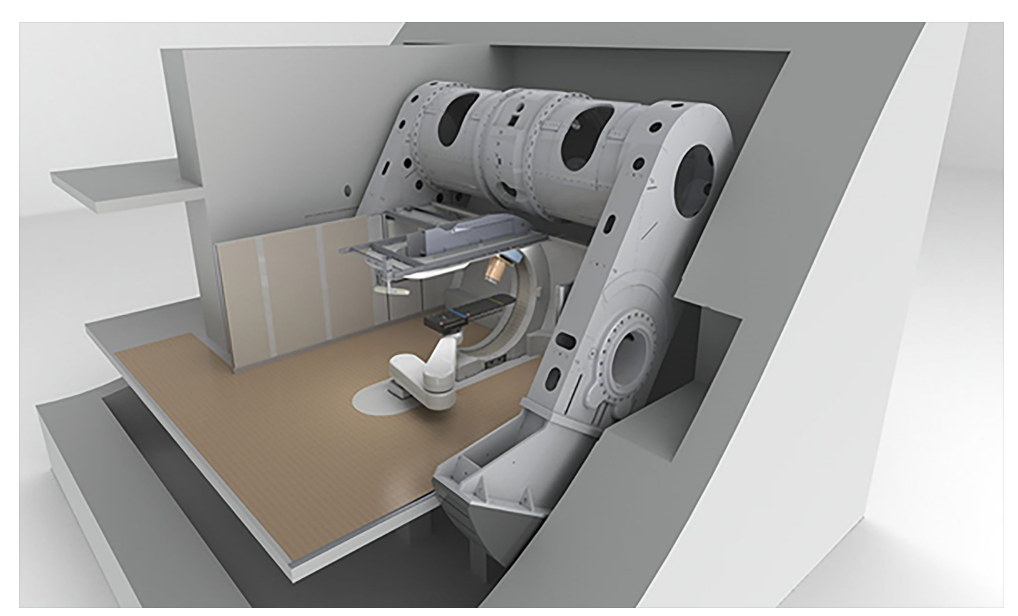

FIGURE 5 | The MEVION S250 gantry-mounted proton synchrocyclotron - after Jongen (7).

\subsubsection{Conventionally Wound HTS Magnets for Carbon Therapy}

In 2013 Toshiba began the development HTS magnets one of its aims being to further reduce the size of heavy-ion-therapy gantries (47). Based on Toshiba's above low-temperaturesuperconducting gantry an HTS gantry was designed and model magnet designed, built, and tested. Selected for the windings was a REBCO HTS tape $4 \mathrm{~mm}$ wide and $0.1 \mathrm{~mm}$ thick. Wound with $820 \mathrm{~m}$ of tape, the magnet consisted of 24 saddle-shaped REBCO coils stacked in four layers and attached to an iron yoke (47). Conduction cooled to about $4 \mathrm{~K}$ by a GM cryocooler the magnet generated a bore field of $1.2 \mathrm{~T}$.

\subsubsection{Canted Cosine Theta Magnets for Carbon Therapy}

As mentioned in Section 5.5 at the Heidelburg Ion-Beam Therapy Center (HIT) the 135 tonnes of normal-conducting magnets are supported by a $21 \mathrm{~m}$ long, $6 \mathrm{~m}$ radius, gantry weighing an additional 515 tonnes. The resulting demand for a smaller lighter gantry called for introduction of superconducting magnets. Numerous such systems furnished with conventionally wound $\cos \theta$ and $\cos (2 \theta)$ dipoles and quadrupoles have been designed and/or constructed, e.g. $(33,46-48)$.. To still further reduce size and weight the CCT winding was introduced. Numerous reports describe the implementation of CCT winding within the context of proton therapy [e.g. $(35,40)]$ but the concept should be applicable to both proton and carbon systems.

Robin et al. described a superconducting magnet string for a compact carbon-ion therapy gantry (49). Their gantry $9.97 \mathrm{~m}$ long and $3.12 \mathrm{~m}$ radius carries two $45^{\circ}$ bending dipoles, numerous quadrupoles, and a $90^{\circ}$ large aperture final bending magnet. In a paper that focused attention on the $5 \mathrm{~T} 90^{\circ}$ final bending magnet the authors pointed out that the CCT concept could be applied to dipoles, quadrupoles, and bent magnets ("toroids"). Winding schemes to produce the correct combination of dipolar and multipolar fields were developed. The magnet layout of Kim et al. (19) is identical to that of Robin et al. (49). This work also focused attention on the large aperture $90^{\circ}$ final bending magnet recognizing that the field to bend $430 \mathrm{MeV} / \mathrm{u}$ carbon-ion beam $(R$ $=6.627 \mathrm{Tm}$ ) to a radius of $1.269 \mathrm{~m}$ is $5.22 \mathrm{~T}$. Sextupole components in the fringe field region of the dipole were removed by adjusting the coil winding in the main body of the dipole.

\subsubsection{Recent Initiatives in Magnets and Gantries}

IFAST (Innovative Fostering of Accelerator Science and Technology, a CERN project) was the subject of a meeting convened by L. Rossi (INFL, Milano) that set out to prepare a proposal dealing with Work Package WP-8 (innovative superconducting magnets (L. Rossi)) and WP-4 (magnet design) (50). The objective of WP-4 included engineering design for the HITRI accelerator magnets (see Section 5.5) and the HITRI gantry magnet.

TABLE 9 | Some properties of the toshiba/NIRS superconducting dipoles (46).

\begin{tabular}{|c|c|c|c|}
\hline Magnet Group & BM1-3 & BM4-6 & BM7-10 \\
\hline Bend angle, degrees & 70 & 70 & 90 \\
\hline Bend Radius, $\rho, \mathrm{m}$ & 2.3 & 2.3 & 2.8 \\
\hline Max Dipole Bore Field, $B, T$ & $2.88^{*}$ & 2.88 & 2.37 \\
\hline
\end{tabular}

*Magnetic rigidity of $430 \mathrm{MeV} / \mathrm{u}$ carbon ions, $M=6.627 \mathrm{Tm}$ (Appendix A2) hence $B=M / \rho=2.88 \mathrm{~T}$. 


\subsubsection{Superconducting Toroidal Gantry for Hadron Therapy}

Bottura, Felcini, et al. (CERN) described the magnetic design of a superconducting gantry in the form of a torus configured for proton or hadron therapy. For the windings of this "GaToroid" both LTS (NbTI) and HTS (REBCO) options were considered. The device eliminates the need for rotating structures and operating at constant current and magnetic field is able to deliver a beam at discrete angles over a wide range of energies, e.g. $70 \mathrm{MeV}$ to $250 \mathrm{MeV}$.

\section{THERAPY SYSTEMS}

\subsection{Magnets and Gantries}

The designers of ion-beam therapy systems are continually striving for improved beam optics, smaller size, and lower cost. Regarding optics, pencil-beam scanning was early introduced by the vendors. Introduction of the alternating gradient CCT magnet with its large momentum acceptance ( $25 \%)$ enabled large energy ranges to be transmitted at fixed field, thus reducing the need for low-AC-loss magnet windings. Therapy systems consisting of large accelerators, beam lines and gantries are large and expensive. Superconductors went a long way towards reducing their size. Another important step was taken by MEVION which, by mounting a compact 17 ton synchrocyclotron on the gantry frame, eliminated the need for a string of quadrupoles and bending dipoles. Although the MEVION could be fitted with the desired pencil-beam scanning system, the fixed $250 \mathrm{MeV}$ of the synchrocyclotron called for graphite energy degraders to vary the bean energy. A potential improvement would be to introduce the MIT-ProNova-designed 5 ton ironless variableenergy (70-230 MeV) synchrocyclotron.

Sections 5.5 through 5.7 have outlined the evolution of magnet systems and gantries from proton to carbon-ion, from normal-conducting to superconducting, from standard $\cos \theta$ and $\cos (2 \theta)$ windings, to tilted double-helix (CCT) windings. We see proton magnet system weights decreasing from an estimated $20 \sim 40$ tons to less than 5 tons. Gantry layouts have been simplified and magnets have been improved, for example: (i) Bontoiu's proton gantry (37) consisted of a string of 36 combined-function superconducting magnets, (ii) Toshiba's carbon-ion gantry consisted of 10 combined-function superconducting magnets arranged in 3 groups for bends of $70^{\circ}$ (3 magnets), $70^{\circ}$ (3 magnets), and $90^{\circ}$ (4 magnets) $(18,33$, 46) (47), (iii) Another Toshiba carbon-ion gantry consisted of just 3 superconducting combined function $90^{\circ}$ bend magnets (48), (iv) substitution of the standard $\cos \theta$ and $\cos (2 \theta)$ windings by tilted double-helix (CCT) windings, for which several advantages have been claimed viz: (a) an order of magnitude reduction in weight, (b) possible gantry size reduction, e.g. diameter $5 \mathrm{~m}$ and length $8.3 \mathrm{~m}$, and (c) rapid scanning (35).

\subsection{Magnet Windings 6.2.1 LTS Windings}

Wan et al. (35) (LBNL) have reviewed the suitability of the wellknown low temperature (LTS) and high temperature (HTS) superconductors for gantry magnet windings and conclude that for their proton gantry application NbTi was the conductor of choice. For the windings of their CCT combined-function magnets Wan et al. selected "SSC-inner" NbTi strand $0.8 \mathrm{~mm}$ in diameter, filament diameter $6 \mu \mathrm{m}$. For one of the magnets the 8 conductors in the two dipole layers will be powered in series and the 26 conductors in the two quadruple layers will be separately powered in series. The suitability of NbTi has also been claimed by others, for example: (i) the 10 magnets of a Toshiba gantry (46) are wound with $0.9 \mathrm{~mm}$ diameter NbTi wire, (ii) the 3 combinedfunction magnets of another Toshiba gantry (48) are wound with $0.92 \mathrm{~mm} \mathrm{NbTi}$ wire twisted to $6+1$ for a final cable diameter including insulation of $3.0 \mathrm{~mm}$; the coil currents were $710 \mathrm{~A}$ (quadrupole) and $920 \mathrm{~A}$ (dipole), (iii) another LBNL magnet (40) was wound with series-connected parallel stacks $(6 \times 1$ and $8 \times 1)$ of $1.6 \mathrm{~mm}$ square insulated insulated NbTi wires.

\subsubsection{LTS and HTS Windings}

With a magnetic rigidity of $6.627 \mathrm{Tm}$ a $430 \mathrm{MeV}$ carbon-ion beam can be bent to a radius of $1 \mathrm{~m}$ by a dipole with a bore field of 6.6 T (although the field-at-winding will be higher than this to an extent that depends on magnet design). Wan et al's (35) review of some of the key properties of the wire-formable LTS and HTS superconductors reminds us that with $4.2 \mathrm{~K}$ upper critical fields $\left(B_{c 2}\right)$ of 10.5-11 T (NbTi), 19-27 T $\left(\mathrm{Nb}_{3} \mathrm{Sn}\right), 9-10 \mathrm{~T}$ $\left(\mathrm{MgB}_{2}\right), 70-75 \mathrm{~T}\left(\mathrm{Bi}_{2} \mathrm{Sr}_{2} \mathrm{CaCu}_{2} \mathrm{O}_{8+\mathrm{x}}\right.$, i.e. “Bi:2212”), and 92-95 T $\left(\mathrm{YBa}_{2} \mathrm{Cu}_{3} \mathrm{O}_{7-\mathrm{x}}\right.$, i.e. "REBCO") they all apparently qualify from a $4.2 \mathrm{~K}$ critical field standpoint. Down-selection then involves consideration of other materials properties, magnet fabrication problems, and cryogenic issues. After winding $(W)$ with $\mathrm{Nb}_{3} \mathrm{Sn}$ wire the magnet needs to be reacted $(R)$ for $\sim 160$ hours at temperatures up to $650^{\circ} \mathrm{C}$ and $\mathrm{Bi}: 2212$ coils require $\sim 125$ hours at $888^{\circ} \mathrm{C} \cdot \mathrm{MgB}_{2}$ requires a relatively mild heat treatment $(60 \mathrm{~min} /$ $675^{\circ} \mathrm{C}$ ) and either $W \mho R$ or $R \& W$ coils can be produced. From a manufacturing standpoint $\mathrm{MgB}_{2}$ is more attractive than $\mathrm{Nb}_{3} \mathrm{Sn}$ and Bi:2212 but its critical field does not leave enough margin to enable conduction cooled operation at the above field strengths. $\mathrm{NbTi}$ and REBCO wires in the as-received condition are suitable for coil winding; this is a great advantage. The selection in favor of REBCO, dictated by cryogenic issues, is discussed in the following sections.

\subsection{Cryogenics}

\subsubsection{Gantry Cooling}

The accelerator magnets of high energy physics are wound with high-current-carrying Rutherford cable to maintain a high ampere-turn ratio while minimizing the magnet inductance. Different considerations govern the choice of magnet design and conductor size in superconducting particle beam gantries. Inductance is not an issue but magnet current has to be relatively small to minimize current-lead heat leak into the cryostat. Since the magnet heat load scales with ramp rate a large momentum acceptance (especially if it allows fixed-field operation) is beneficial for thermal design (40). Because the gantry needs to rotate, magnet cooling by liquid helium is not feasible. Instead all superconducting gantry systems are cryogen-free, conduction cooled by way of high conductivity links to cryocooler cold heads. Numerous cold heads may be connected in parallel to 
various parts of the magnet system (35). The final $90^{\circ}$ dipole of a joint IBA/CEA Saclay study was cooled by 10 Sumitomo cryocoolers (18). Mounted on the Toshiba heavy-ion gantry referred to above (47) are 3 groups of 10 bending magnets, BM1-3, BM4-6 and BM7-10. They are conduction cooled with $1.5 \mathrm{~W} / 4.2 \mathrm{~K}$ Gifford-McMahon cryocoolers - three sets on each of BM1-6 and four sets on each of BM7-10 for a total of 34 cryocoolers (47). At a Sumitomo (SHI) model RDK-4150 $(1.5 \mathrm{~W} / 4.2 \mathrm{~K})$ cold head weight of $18.5 \mathrm{~kg}(40.8 \mathrm{lb}) 34$ cryocoolers would contribute about $3 / 4$ ton to the weight of the gantry. A lighter gantry could be enabled by circulating gaseous or supercritical helium through the magnets from an off-gantrymounted cooling and pumping system (51-54) or by implementing a cryogenic oscillating heat pipe system (55).

\subsubsection{The Choice of REBCO Conductor for Cryogen- Free Carbon-Ion Gantries}

In 2016 Iwata et al. and others from the Toshiba Corporation reported on the design of a compact gantry for $430 \mathrm{MeV} / \mathrm{u}$ carbon ions (48). Each of the three $90^{\circ}$ dipoles was to have a bend radius of $1.32 \mathrm{~m}$ and hence a dipole field strength of $5.02 \mathrm{~T}$. The results of the design study did not include cryogenics and provided no indication of the feasibility of maintaining a field of $5 \mathrm{~T}$ under cryogen-free conditions. On the other hand the Toshiba group did report on the design $(46,47)$, test results $(47)$, and delivery to NIRS of a heavy ion rotating gantry. As reported above (section 5.7.2, Table 9) the three sets of NbTi-wound bending magnets had maximum fields of $2.88 \mathrm{~T}, 2.88 \mathrm{~T}$, and 2.37 T. It is doubtful if fields much higher than this can be achieved in commercial conduction-cooled systems. Attempts to build NbTi-wound conduction-cooled magnets for magnetic resonance imaging (MRI) have not succeeded, while recent research and development of conduction-cooled $3 \mathrm{~T}$ MRI systems has focused on $\mathrm{MgB}_{2}$. But for ion-beam therapy, to avoid the difficulties associated with reaction-heat-treatment and to gain the advantages of its high $T_{c}, B_{c 2}$, and engineering critical current density, $J_{E}$, and cryogenic stability, REBCO is arguably the conductor of choice.

The $4.2 \mathrm{~K}, 5 \mathrm{~T}$ the $J_{E}$ of "standard" SuperPower REBCO tape at $4.5 \times 10^{3} \mathrm{~A} / \mathrm{mm}^{2}(\mathrm{~B} / /)(56)$ is 4 times greater than that of NbTi. An even higher $J_{E}\left(5.4 \times 10^{4} \mathrm{~A} / \mathrm{mm}^{2}\right)$ is becoming obtainable as a result of: (i) increasing the $\mathrm{Zr}$ doping content from $7.5 \%$ to $25 \%$ (x3), (ii) increasing the film thickness (x2), (iii) reducing the tape thickness from $100 \mu \mathrm{m}$ to $50 \mu \mathrm{m}(\mathrm{x} 2)$. As a result the $4.2 \mathrm{~K}, 5 \mathrm{~T} J_{E}$ of REBCO tape is more than 40 times that of $\mathrm{NbTi}$. One reason for the rejection of REBCO was that tape geometry was not regarded as suitable for magnet winding. This is no longer a problem. REBCO is now available in wire form - thin tapes spirally wrapped along a wire core $0.51-0.8 \mathrm{~mm}$ in diameter.

\section{PROSPECTS FOR IMPROVEMENT}

\subsection{Need for Image Guidance}

To perform an efficient treatment accurate image guidance techniques that can distinguish a tumor from healthy tissue are required. Imaging is associated with target accuracy, of targeting, dosimetry, treatment outcome assessment, and prognosis. Images are used in radiation therapy during the following processes: (1) offline treatment planning - tumors and other anatomical structures are identified and the treatment is simulated on a treatment planning computer. During this process CT, MRI and even PET-CT or other type of images are commonly used; (2) imaging is applied to set up the patient in the treatment room and to convert the virtual treatment (a treatment plan) into the real treatment; (3) images are used to assess the treatment outcome: effectiveness, toxicities, and prognosis, etc. While Processes 1 and 3 are usually carried out offline, Process 2 is often performed with the imaging device attached to the treatment machine inside the treatment room to align the patient in the treatment position or during the treatment to monitor the targeting of tumors and organs-atrisk. Process 2 is complex and needs to conform to the gantry and the beam delivery system. The currently available imageguided particle therapy systems are mainly based on $2 \mathrm{D}$ orthogonal X-ray imaging, in-room 3D computed tomography (CT) or on-board cone-beam CT (CBCT) imaging. However, 3D or $4 \mathrm{D} \mathrm{CT}$ and MRI images are commonly used off-line for treatment planning (57). Imaging technologies such as the proton Computed Tomography (or pCT) $(58,59)$ or MRguided proton therapy have been proposed but are not yet available for clinical use (60). While X-ray based imaging has difficulty resolving soft-tissue the converse is true for MRIguided particle therapy. MRI guidance in photon (radiation) therapy MRgRT, which entered clinical practice not long ago, gradually became popular during the past decade (61). Currently there are several commercial available MRgRT manufacturers in the market: ViewRay (62), Elekta (63), and Varian Medical Systems/IMRIS (64).

In order to take advantage of the peak effect (discussed in Section 2.1) the exact depth of the tumor (the target) must figure into the treatment. An error in the target depth of a few $\mathrm{mm}$ that may result in only a few percent change in the photon dose may lead to a $100 \%$ change in the proton dose (67). This emphasizes the need for direct visualization of the tumor position. Such "image guidance" using MRI has been proposed and modeled by numerous researchers (see section 7.3). The principle of MRI guided proton therapy is illustrated in Figure 6.

\subsection{MRI and Proton Therapy}

Photon irradiation requires a well-defined beam aimed in the right direction. In proton therapy it is also necessary to predict ensure that the beam will terminate at the tumor side. The additional margin needed for range uncertainty detracts from the benefit of proton therapy. Image guidance is introduced to overcome this uncertainty (67). In a combined MRI-proton system it is necessary to consider mutual electromagnetic interaction between the proton beam and the MRI field. For example Monte Carlo computer simulations and measurements have revealed that a $190 \mathrm{MeV}$ proton beam would be deflected by about $1 \mathrm{~cm}$ upon entering a $1 \mathrm{~T}$ field. Such effects would influence treatment planning and dose delivery. External magnetic fields that could influence MR image quality come 


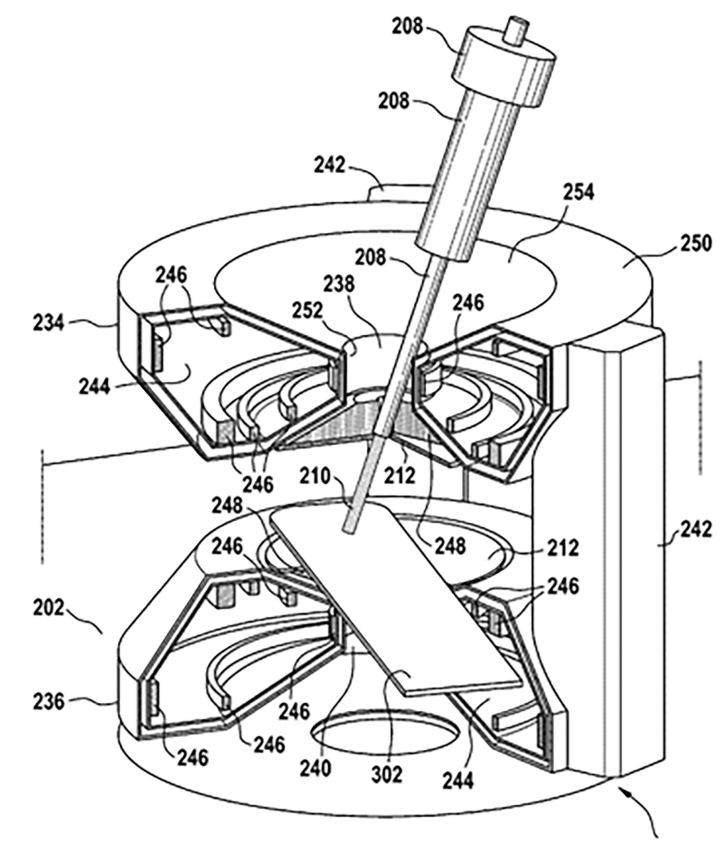

FIGURE 6 | Possible configuration of a hybrid MRI proton system, taken from patent application US 10 , 173 , 077 B2 (45). Date of Patent: Jan. 8, 2019 (67).

from sources such as: (i) the proton generating cyclotron, (ii) the gantry beam line and steering magnets; the fringe field of such magnets, which can be up to $100 \mu \mathrm{T}$, could detrimentally affect the MR image quality. To further investigate such effects Hoffmann and his team $(65,66)$, in association with OncoRay, attached an open $0.22 \mathrm{~T}$ MRI scanner to a fixed horizontally mounted proton research beam line, Figure 7 .

In a useful experimental study Inaniwa et al. (68) investigated the effects of magnetic fields $(0.3$ and $0.6 \mathrm{~T})$ applied transversely $\left(B_{T}\right)$ and longitudinally $\left(B_{L}\right)$ a proton beam adjusted to deposit energies of 1.1 and $3.3 \mathrm{keV} / \mu \mathrm{m}$ into normal and cancer cells. Effectiveness was gauged by the index $R_{10} \equiv D_{W O} / D_{W}$ which is the ratio of the dose that would result in a survival fraction of $10 \%$ in the absence $\left(D_{W O}\right)$ and presence $\left(D_{W}\right)$ of the magnetic field.

For cancer cells exposed to $1.1 \mathrm{keV}(3.3 \mathrm{keV})$ proton beams $R_{10}$ increased to 1.10 (1.11) and 1.11 (1.12) in longitudinal magnetic fields $\left(B_{L}\right)$ of $0.3 \mathrm{~T}(0.6 \mathrm{~T})$.

For normal cells $R_{10}$ increased to 1.13 (1.17) and 1.17 (1.30) in these longitudinal magnetic fields.

For both normal and cancer cells $R_{10}$ showed no significant change in the transversely applied fields

Inaniwa et al. pointed out that the longitudinal field enhancement effect should be taken into account in the design of an MRI-proton system (68).

\subsection{Experiment and Modeling}

No commercial scale versions of the research system described in Figure 7 can be made available for experimentation. However numerous modeling studies have been undertaken on the general topic of beam-line/magnetic-field interactions (see Table 10, Bibliography). Oborn et al. (69) have modeled the path of a proton beam through the $3 \mathrm{D}$ field of a $1 \mathrm{~T}$ split bore MRI magnet. Used for the modeling was the software GEANT4 (GEometry ANd Tracking), a platform for simulating the passage of charged particles through matter using a Monte Carlo method. Significant rotation of the beam was observed in the longitudinal orientation while a more complex path was seen in the transverse field. It was concluded that pencil beam scanning was favored for either longitudinal or transverse field orientations.

\subsection{Recommendations}

MRI guidance should be introduced in order take advantage of proton over photon therapy. In so doing the influence of external magnetic fields emanating from the imaging and beam guidance systems on image quality needs to be investigated. In so doing it would be useful to produce an engineering design for a full-scale proton-beam/MRI system (e.g. Figure 6) using GEANT-MonteCarlo method modeling. In addition (or alternatively) a smallscale experimental prototype MRI-integrated proton therapy system (e.g. Figure 7) could be constructed and used for investigating beam-line/magnetic-field interactions. Given that longitudinal field dose enhancement and other such effects exist, experiments using such a system could be designed to investigate the underlying mechanisms.

To build an effective image-guided or MR-guided proton or particle therapy system, a comprehensive and sophisticated design of accelerator, beam-line, gantry, magnets, and the imaging components needs to be carefully calculated so that all sub-systems could be integrated into a unit that operates efficiently and meets the clinical requirements while reducing cost to a minimum.

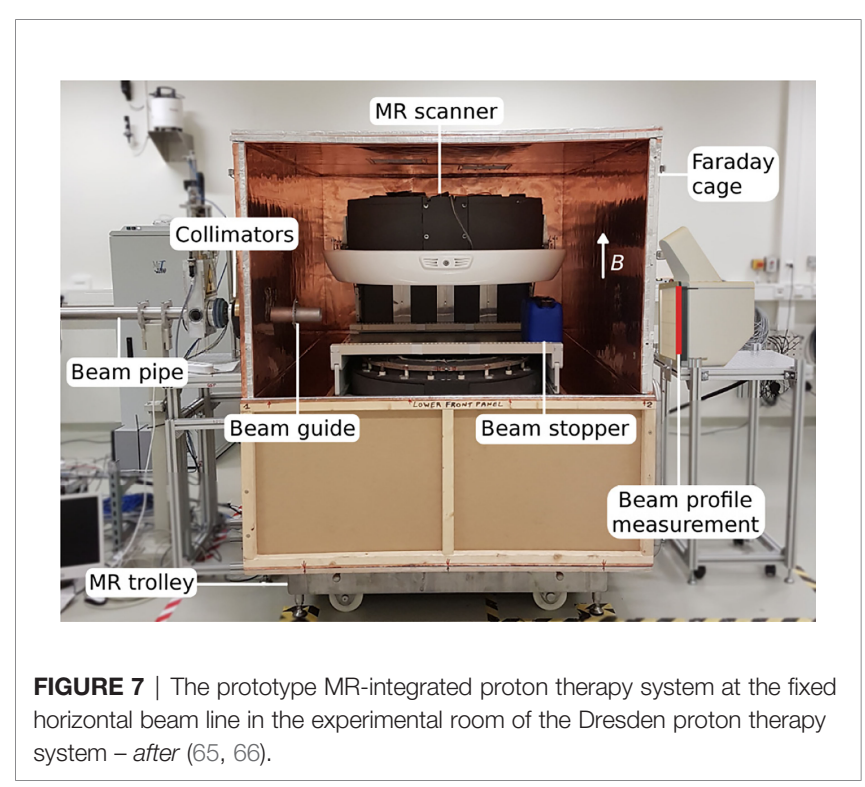


TABLE 10 | Bibliography on MRI-Guided Ion-Beam Therapy.

B.M. Oborn, S. Dowdel, P.E. Metcalf, et al., "Future of Medical Physics: Real-time MRI-guided proton therapy" Med. Phys. 44 77-90 (2017)

L.N. Burigo nd B.M. Oborn, "MRl-guided proton therapy planning: accounting for an inline MRI fringe field", Phys Met Bio/ 64215015 (2019)

A. Hoffmann, B. Oborn, M. Moteabbed, et al, "MRl-guided proton therapy: a review and a preview", Radiat Oncol 15, 129 (2020)

T. Freeman, "MRI-guided proton therapy: a status update", physics world (2019)

S.E.M. Huijsse, A. Knopf, L.V. van Dijk, et al "MR-only guided proton therapy: advances, future perspectives and challenges", MReadings:MR in RT, pp. 16-21

B.M. Oborn, S. Dowdell, P.E. Metcalf, et al, "MRI Guided Proton Therapy: Pencil beam scanning in an MRI fringe field", Radiother Oncol. 118(Supplement 1):S78-9

(2016)

B.M. Oborn, S. Dowdell, P.E. Metcalf, et al,"Proton beam deflection in MRI fields: Implications for MRI-guided proton therapy: Medical Physics 42, 2113 (2015)

B.M. Oborn, S. Dowdell, P.E. Metcalf, et al,"Future of Medical Physics: Real-time MRI guided Proton Therapy", Med Phys. 44 77-90 (2017)

M. Moteabbed, J. Schuemann, and H. Paganetti", Dosimetric feasibility of real-time MRI-guided proton therapy" Medical Physics, 41 111713 (11pp) (2014)

$\mathrm{J}$ Hartman, C Kontaxis, G H Bol, et al. "Dosimetric feasibility of intensity modulated proton therapy in a transverse magnetic field of 1.5 t." Physics in Medicine and

Biology, 605955 (2015)

J. Hartman, J.J.W. Lagendijk, B.W. Raaymakers, et al., "MRI guided proton therapy", UMC Utrecht

B.W. Raaymakers, A.J. Raaijmakers, and J.J. Lagendijk, "Feasibility of MRI guided proton therapy: magnetic field dose effects", Phys Med Biol. 53 5615-22 (2008)

C. Kurz, G. Landry, A.F. Resch et al., "A Monte-Carlo study to assess the effect of 1.5 T magnetic fields on the overall robustness of pencil-beam scanning proton radiotherapy plans for prostate cancer", Phys Med Biol. 62 8470-8 (2017)

H. Fuchs, P. Moser, M. Gröschl, et al., "Magnetic field effects on particle beams and their implications for dose calculation in MR-guided particle therapy", Med Phys. 44 1149-56 (2017)

S.M. Schellhammer and A.L. Hoffmann, "Prediction and compensation of magnetic beam deflection in MR-integrated proton therapy: a method optimized regarding accuracy, versatility and speed", Phys Med Biol. 62 1548-64 (2017)

S.M. Schellhammer, A.L. Hoffmann, S. Gantz, et al. "Integrating a low-field open MR scanner with a static proton research beam line: proof of concept", Phys Med Biol $6323 \mathrm{LTO1}(2018)$

C.M. Rank, N. Hünemohr, A.M. Nagel, et al., "MRI-based simulation of treatment plans for ion therapy in the brain region", Radiother Oncol. 109 414-8 (2013)

R. Wolf and T. Bortfeld, "An analytical solution to proton bragg peak deflection in a magnetic field", Med Phys 57 N329-N337 (2012)

S.M. Schellhammer, A.L. Hoffmann, S. Gantz, et al., Integrating a low-field open MR scanner with a static proton research beam line: proof of concept”, Phys Med Biol. 63 LT01 (2018)

G.G. Marmitt, M. van Goethem, M. Meijers, et al., "Experimental validation of magnetic field deflections of proton beams for online MR-guided PT", 6th MR in RT Symposium, Utrecht, July $1^{\text {st }}-3^{\text {rd }}(2018)$

R. Wolf and T. Bortfeld, "An analytical solution to proton bragg peak deflection in a magnetic field," Phys. Med. Biol. 57(17), N329-N337

T. Inaniwa, M. Suzuki, S. Sato, et al. "Effect of external magnetic fields on biological effectiveness of protonbeams", Int. J. Radiation Oncology. Biology. Physics : BIOLOGY CONTRIBUTION, 106 597-603 (2020)

\section{SUMMARY}

The paper begins by emphasizing the clinical and commercial importance of proton and carbon ion (in general, hadron) therapy and refers to the manufacturers of such systems of which more than 120 are installed or are under construction worldwide. A general review of charged particle therapy systems refers to six manufacturers and provides in tabular form some details of systems installed in the US, Europe, Asia, and elsewhere. The principles of particle beam therapy are described in terms of the Bragg peak and the spread-out Bragg peak (SOBP) and a comparison is made of the therapeutic properties of photons (x-rays) versus proton-beams and the latter versus carbon-ions beams. An introduction to particlebeam acceleration is followed by descriptions of normalconducting and superconducting (SC) cyclotrons (including the isosynchronous cyclotron and the synchrocyclotron) and of synchrotrons. An interesting case study describes the evolution of a normal-conducting 220 ton cyclotron into an iron-free SC synchrocyclotron weighing only 5 tons.

The principles of gantry design and the components of the magnet string are outlined. Particle-beam guidance is described in terms of the beam's magnetic rigidity and the relationship of bend radius, $\rho$, to bending-dipole field strength, $B$. The development of the needed SOBP requires a range of particlebeam energies, typically proton energies of 70-240 $\mathrm{MeV}$. The associated rapid field-sweep would be difficult for SC dipoles unless dipole design allows for broad momentum acceptance. This statement introduces a detailed discussion of gantry magnets for both proton therapy and carbon-ion therapy.

First to be described is the use of normal-conducting ironcore gantry magnets whose $1.8 \mathrm{~T}$ bore field calls for bend radii of $1.35 \mathrm{~m}$ (250 MeV protons) and $3.65 \mathrm{~m}$ (425 MeV/u carbon). Thus although proton gantries are adequately served by such magnets their use in a one-of-a-kind carbon gantry has led to an unacceptably large structure. Both proton and carbon gantries benefit from the use of SC magnets. The "direct replacing" of normal-conducting magnets with SC ones is expected to lower the weight and cost of the gantry.

Several winding arrangements are described. Conventional windings are the familiar $\cos \theta$ dipolar and $\cos 2 \theta$ quadrupolar windings of high energy particle physics. In the present context they have given rise to the combined-function winding in which a $\cos 2 \theta$ quadrupole coil is wound on top of a $\cos \theta$ dipole coil. Such magnets possess much larger momentum acceptances than do individual dipoles and quadrupoles and enable beams of a wide range of energies to be controlled by only a few field steps. The use of conventionally wound SC magnets in both proton and hadron therapy is reviewed. Then an alternative winding, the tilted double helix now referred to as the canted cosine theta (CCT) winding, is introduced A combined-function version of it is produced by winding a $\cos \theta$ dipole on top of a $\cos 2 \theta$ quadrupole. Next to be described is a new magnet concept the alternating-gradient CCT (AGCCT) - in which the inner 
quadrupole consists of multiple sections with the current reversed between sections. This design can be characterized as fixed-field alternating-gradient since its large momentum acceptance enables a large energy range to be transmitted without changing the field. Finally, mention is made of a novel CERN-designed superconducting toroidal gantry for hadron therapy, GaToroid. This device, operating under steady state current and magnetic field, is able to deliver a beam at discrete angles over a range of treatment energies.

Cooling of SC rotating gantry magnets is an important engineering task. Cooling by liquid helium is not feasible. Instead some form of liquid-cryogen-free or conduction cooling is required. Some systems make use of locally mounted Gifford-McMahon cryocoolers (typically $1.5 \mathrm{~W} / 4.2 \mathrm{~K}$ each), other suggested cooling modes involve the circulation of gaseous or supercritical helium or the use of a cryogenic oscillating heat pipes.

The choice of superconductor for gantry-magnet winding is discussed in detail. Numerous proton magnets have been wound with NbTi wires - individual 0.8-0.9 mm diameter wires, a $(6+$ 1)-wire twisted cable, parallel stacks of $1.6 \mathrm{~mm}$ square insulated wires. A high-temperature-superconducting (HTS) magnet (bore field $1.2 \mathrm{~T}$ ), conventionally wound with REBCO tape (4 $\mathrm{mm} \mathrm{x}$ $0.1 \mathrm{~mm}$ ), has been designed built and tested. In general the bore fields, even of SC magnets, have been relatively low, often less than $3 \mathrm{~T}$. But for future carbon-ion systems, especially when gantry size and hence dipole bend radius is to be minimized, bore fields of order $6 \mathrm{~T}$ will be needed. To satisfy this requirement in a cryogen-free magnet superconductors with critical temperatures and critical fields higher than those of $\mathrm{NbTi}$ will be needed. A review of the properties of several low-temperature- and high temperature superconductors indicates that REBCO is the material of choice - not in the form of a difficult-to-wind tape but as a wire (or cable) made from narrow tapes spirally wrapped along a thin wire core.

\section{REFERENCES}

1. Goethals P-E, Zimmermann R. Proton Therapy World Market Report Edition 2015. Louvain-la-Neuve, Belgium: MEDraysintell (2015).

2. P-E Goethals, R Zimmermann eds. Proton Therapy World Market Report \& Directory - Edition 2015. Louvain-la-Neuve, Belgium: MEDraysintell (2016).

3. Available at:protonbob.com/about-proton-therapy/.

4. Available at: www.proton-therapy-canters.com.

5. Norimine T, Umezawa M, Hiramoto K. A Design of a Rotating Gantry With Easy Steering for Proton Therapy. In: Proc. European Particle Accelerator Conference (EPAC 2002). Paris, France: JACOW, (2002). p. 2751-3.

6. Goitein M, Lomax AJ, Pedroni ES. Treating Cancer With Protons. Phys Today (2002) 55:45. doi: 10.1063/1.1522215

7. Jongen Y. Review on Cyclotrons for Cancer Therapy. In: Proc. CYCLOTRONS. Lanzhou, China: JACOW. (2010). p. 398-403.

8. Wilson RR. The Radiological Use of Fast Protons. Radiology (1946-47) 47:487-491. doi: 10.1148/47.5.487

9. Newhauser W, Zhang R. The Physics of Proton Therapy. Phys Med Bio (2016) 60:R155-209. doi: 10.1088/0031-9155/60/8/R155

10. Available at: http://www.mitsubishielectric.com/bu/particlebeam/ installations/installations.html\#list04.

11. Prestemon S. Superconducting Magnets for Medical Accelerators. In: ARIES Collaboration Meeting. Budapest (2019). Available at: https://indico.cern.ch/
Finally, an important "Prospect for Improvement" would be the introduction into the system of MRI image guidance. In order to take advantage of the Bragg peak effect the exact targeting of the tumor and positioning of the patient must figure into the treatment. The charged-particle beam has to be guided by image visualization using X-ray, CT, and hopefully MRI. An error in the target depth of a few $\mathrm{mm}$ that may result in only a few percent change in photon dose may lead to a $100 \%$ change in the proton dose (67). Unlike MRI-guided photon therapy the direct interaction of the magnetic field with the charged particle beam presents a huge challenge such that MRI image-guided proton/particle therapy has not yet been available in clinical practice. Modeling studies have been undertaken on the general topic of beam-line/magnetic field interaction using, for example, the software GEANT4 (GEometry And Tracking) a platform for simulating the passage of charged particles through matter using a Monte Carlo method. The paper concludes by noting that a comprehensive design of accelerators, gantries, magnets and imaging systems for particle beam therapy would be the best way to produce the most efficient and cost effective particle-beam therapy system.

\section{AUTHOR CONTRIBUTIONS}

EC wrote the first draft of the manuscript. LL and NG wrote sections of the manuscript. All authors contributed to manuscript revision, read, and approved the submitted version.

\section{FUNDING}

This work was supported by the National Institute of Biomedical Imaging and Bioengineering, under grant R01EB018363.

event/756346/contributions/3324788/attachments/1827595/2991555/ superconducting_magnets_for_medical-Prestemon-final.pdf.

12. Rossi L. HITRI+ and I-FAST: Next Eu Programs for SC Heavy Ion Therapy Machine. In: HTRI+ and I.FAST WP8-Magnets for HT (2020). Available at: https://indico.cern. ch/event/977607/contributions/4117564/attachments/2156130/3636848/HITRI\% 20and\%20IFAST\%20WP8\%20pre-start\%20meeting1-2020-12-03.pdf.

13. Levin WP, Kooy H, Loeffler JS, DeLaney TF. Proton Beam Therapy. $\mathrm{Br} J$ Cancer (2005) 93:849-54. doi: 10.1038/sj.bjc.6602754

14. Available at: japan-product.com/ads/saga-himat-heavy-ion-cancertreatment-center/.

15. Tsujii H. "Overview of Carbon-Ion Radiotherapy", Micro, Mini, and Nanodosimetry Conference 2016 (MMND\&ITRO2016) IOP Conf. Series: J Phys Conf Ser (2017) 777 012032:4 pp. doi: 10.1088/1742-6596/777/1/012032

16. Tsujii H, Kamada T, Shirai T, Noda K, Tsuji H, Karasawa K, et al. Carbon-Ion Radiotherapy; Principles, Practices and Treatment Planning. Japan: SpringerVerlag (2014).

17. Castro JR, Saunders WM, Tobias CA, Char D, Phillips TL, Alpen EL, et al. Treatment of Cancer With Heavy Charged Particles. Int J Radiat Oncol Biol Phys (1982) 8(12):2191-8. doi: 10.1016/0360-3016(82)90569-7

18. Alonso JR, Antaya TA. Superconductivity in Medicine. Rev Accelerator Sci Technol (2012) 5:227-63. doi: 10.1142/S1793626812300095

19. Kim J, Yoon M. Design Study of a Superconducting Gantry for Carbon Beam Therapy. J Korean Phys Soc (2016) 69:1048-52. doi: 10.3938/jkps.69.1048. 
20. Owen H, Holder D, Alonso J, Mackay R. Technologies for Delivery of Proton and Ion Beams for Radiotherapy. Int J Mod Phys A (2014) 29(14):1441002. doi: 10.1142/S0217751X14410024

21. Sauer A, Deitinghoff H, Klein H. Beam Dynamics Studies for a High Current Ion Injector, in: XX International Linac Conference, Monterey, CA: JACOW, August 21-25, 2000. pp. 803-5.

22. Lombardi AM. The Radio Frequency Quadrupole (RFQ) . Available at: https:// cds.cern.ch/record/1005049/files/p201.pdf.

23. Caliskan A, Ylmaz M. DTL Cavity Design and Beam Dynamics for a TAC Linear Proton Accelerator. Chin Phys C (2012) 36:167. doi: 10.1088/1674$1137 / 36 / 2 / 012$

24. Mandrillon P. Injection Into Cyclotrons, CERN Accelerator School, CERN, . Available at: https://cds.cern.ch/record/399426/files/p153.pdf.

25. Blosser H, Bailey J, Burleigh R, Johnson D, Kashy E, Kuo T, et al. Superconducting Cyclotron for Medical Application. IEEE Trans Magnetics (1989) 25:1746-54. doi: 10.1109/20.92639

26. Pearson E, Abs M, Henrotin S, Kleeven W, Van de Walle J, Verbruggen P, et al. The New IBA Superconducting Synchrocyclotron (S2C2) From Modelling to Reality. Joint Universities Accelerator School, CERN. Available at: https:// indico.cern.ch/event/683638/contributions/2801891/attachments/1563524/ 2558991/Part-3.pdf.

27. Blosser H, Burleigh R, Johnson D, Kuo T, Marti F, Vincent J, et al. (1989). Medical Accelerator Projects at Michigan State University, in: Proc. IEEE Particle Accelerator Conf, Accelerator Science and Technology, Chicago, IL, 1989. p. 5 pp.

28. Available at: https://tlo.mit.edu/technologies/ironless-cyclotron.

29. Li H, Sahoo N, Poenisch F, Suzuki K, Li Y, Li X, et al. Use of Treatment Log Files in Spot Scanning Proton Therapy as Part of Patient-Specific Quality Assurance. Med Phys (2013) 40 021703:12 pp. doi: 10.1118/1.4773312

30. Pavlovic M. Beam-Optics Study of the Gantry Beam Delivery System for Light-Ion Cancer Therapy. Nucl Instrum Methods Phys Res A (1997) 399:43954. doi: 10.1016/S0168-9002(97)00939-X

31. Flanz JB. Large Medical Gantries. Proc IEEE Particle Accelerator Conf (1995) 3:2004-8. doi: 10.1109/PAC.1995.505434

32. Derenchuk L. The ProNova SC360 Gantry. Modern Hadron Therapy Gantry Developments 16 Jan to 17 Jan 2014 (2014) Cockcroft Institute, Daresbury UK, available at: http://indico.hep.manchester.ac.uk/getFile.py/access?contribId= 27\&resId $=0$ \&materialId $=$ slides\&confId $=4226$

33. Iwata Y, Fujita T, Furukawa T, Hara Y, Matsuba S, Mizushima K, et al. Superconducting Gantry for Carbon-Ion Radiotherapy, 9th International Particle Accelerator Conference IPAC2018, Vancouver, BC, Canada: JACoW Publishing. doi: 10.18429/JACoW-IPAC2018-TUZGBF1

34. Available at: www.proton-cancer-treatment.com/proton-center/treatmenttechnology.../nozzles/.

35. WanW, BrouwerL, CaspiS, PrestemonS, GerbershagenA, SchippersJM, RobinD. Alternating-Gradient Canted Cosine Theta Superconducting Magnets for Future Compact Proton Gantries. Phys Rev S.T Accel Beams (2015) 18 103501:23. doi: 10.1103/PhysRevSTAB.18.103501

36. Bajard M, Kircher F. (2008). Rotative Gantry for Dose Distribution in Hadron Therapy, in: Proc. European Particle Accelerator Conference (EPAC08), Genoa, Italy. pp. 1779-81.

37. Bontoiu C, Sanchez-Segovia J. (2015). Design of a Superconducting Gantry for Protons, in: Proc. International Particle Conference (IPAC2015), Richmond, VA, USA. pp. 2268-70.

38. Wan W, Robin D, Sessler A, Sun C. (2012). Compact Gantry With Large Energy Acceptance, in: Proc. International Particle Accelerator Conference (IPAC2012), New Orleans, LA, USA. pp. 4100-2.

39. Meyer DI, Flasck R. A New Configuration for a Dipole Magnet for Use in High Energy Physics Application. Nucl Instrum Methods (1970) 80:339-41. doi: 10.1016/0029-554X(70)90784-6

40. Brouwer L, Caspi S, Hafalia R, Hodgkinson A, Prestemon S, Robin D, et al. Design of an Achromatic Superconducting Magnet for a Proton Therapy Gantry. IEEE Trans Appl Supercond. (2017) 27 4400106:6 pp. doi: 10.1109/ TASC.2016.2628305

41. Koschik A, Bula C, Dippich J, Gerbershagen A, Grossmann M, Schippers JM, et al. Gantry 3: Further Development of the PSI PROSCAN Therapy Facility. In: Proc. International Particle Accelerator Conference ( Ipac2015). Richmond, VA, USA (2015). p. 2275-7.
42. Umezawa M, Ebina F, Fujii Y, Matsuda K, Hiramoto K, Umegaki K, et al. Development of Compact Proton Beam Therapy System for Moving Organs. Hitachi Rev (2015) 64:506-13.

43. Available at: https://www.blogs.va.gov/.../va-and-stanford-to-pursue-thenations-first-hadron-cente.

44. Available at: https://source.colostate.edu/plan-presents-new-hope-for-uscancer-patients/.

45. Kramer D. Carbon-Ion Cancer Therapy Shows Promise. Phys Today (2015) 68(6):24-5. doi: 10.1063/PT.3.2812

46. Iwata Y, Noda K, Shirai T, Murukami T, Furukawa T, Mori S, et al. Design of a Superconducting Rotating Gantry for Heavy-Ion Therapy. Phys Rev S.T. Accel Beams (2012) 15 044701:14 pp. doi: 10.1103/PhysRevSTAB.15.044701

47. Takayama S, Koyanagi K, Miyazaki H, Takimi S, Orikasa T, Ishii Y, et al. Design and Test Results of Superconducting Magnet for Heavy-Ion Rotating Gantry. J Physics: Conf Ser (2017) 871 012083:8 pp. doi: 10.1088/1742-6596/871/1/012083

48. Iwata Y, Shirai T, Noda K. Design of Superconducting Magnets for a Compact Carbon Gantry. IEEE Trans Appl Supercond. (2016) 26 4400104:4 pp. doi: 10.1109/TASC.2015.2509187

49. Robin DS, Arbelaez D, Caspi S, Sun C, Sessler A, Wan. and M. Yoon W. Superconducting Toroidal Combined-Function Magnet for a Compact Ion Beam Cancer Therapy Gantry. Nucl Instrum Methods Phys Res A (2011) 659:484-93. doi: 10.1016/j.nima.2011.08.049

50. Rossi L. Meeting on I_FAST-WP8 ISM With Labs and Industry. Available at: https:/indico.cern.ch/event/878686/contributions/3717262/attachments/ 1973594/3283832/I-FAST_-_WP8_ISM_CCT_-_discussion_2020-01-22_ ROSSI-V2.pdf.

51. Takahashi M, Iwai S, Miyazaki H, Tosaka T, Tasaki K, Hanai S, et al. Design and Test Results of a Cryogenic Cooling System for a 25-T Cryogen-Free Superconducting Magnet. IEEE Trans Appl Supercond. (2017) 27 4603805:5 pp. doi: 10.1109/TASC.2017.2673762

52. Green MA. Cooling Intrinsically Stable Superconducting Magnets With Supercriical Helium. IEEE Trans Nucl Sci (1971) 18:669-73.

53. Green MA, Burns WA, Taylor JD. Forced Two-Phase Helium Cooling of Large Superconducting Magnets. Adv Cryo Eng (1980) 25:420-30. doi: 10.1007/978-1-4613-9856-1_51

54. Eberhard PH, Gibson GA, Green MA, Rose RR, Smits RG, Taylor JD. Two Phase Cooling for Superconducting Magnets". Adv Cryo Eng (1986) 31:70914. doi: 10.1007/978-1-4613-2213-9_80

55. Natsumi K, Mito T, Yanagi N, Tamura H, Tamada T, Shikimachi K, et al. Development of Cryogenic Oscillating Heat Pipe as a New Device for Indirect/ Conduction Cooled Superconducting Magnets. IEEE Trans Appl Supercond. (2012) 22 4703904:4 pp. doi: 10.1109/TASC.2012.2185029

56. Lee PJ. Available at: http://fs.magnet.fsu.edu/ lee/plot/plot.htm.

57. MacKay RI. Image Guidance for Proton Therapy. Clin Oncol (2018) 30:293-8. doi: 10.1016/j.clon.2018.02.004

58. Li T, Liang Z, Singanallur JV, Satogata TJ, Williams DC, Schulte RW. Reconstruction for Proton Computed Tomography by Tracing Proton Trajectories: A Monte Carlo Study. Med Phys (2006) 33:669-706. doi: $10.1118 / 1.2171507$

59. Esposito M, Waltham C, Taylor JT, Manger S, Phoenix B, Price T, et al. PraVDA: The First Solid-State System for Proton Computed Tomography. Physica Med (2018) 55:149-54. doi: 10.1016/j.ejmp.2018.10.020

60. Hoffmann A, Oborn B, Moteabbed M, Yan S, Bortfeld T, Knopf A, et al. MRGuided Proton Therapy: A Review and a Preview. Radiat Encology (2020) 15 129:13pp. doi: 10.1186/s13014-020-01571-x

61. Otazo R, Lambin P, Pignol J-P, Ladd ME, Schlemer H-P, Baumann M, et al. MRI-Guided Radiation Therapy: An Emerging Paradigm in Adaptive Radiation Encology. Radiology (2020) 298:248-60. doi: 10.1148/ radiol.2020202747

62. Chuong MD, Btyant J, Mittauer KE, Hall M, Lotecha R, Alverez D, et al. Ablative 5-Fraction Stereotactic Magnetic Resonance-Guided Radiation Therapy (MRgRT) With on-Table Adaptive Replanning and Elective Nodal Irradiation for Inoperable Pancreas Cancer. Pract Radiat Oncol (2021) 11:134-7. doi: 10.1016/j.prro.2020.09.005

63. Snyder JE, St Aubin J, Yaddanapudi S, Boczkowski A, Dunkerley DAP, Graves SA, et al. Commisioning of a 1.5 T Elekta Unity MR-Linac: A Single Institution Experience. J Appl Clin Med Phys (2020) 21:160-72. doi: 10.1002/acm2.12902 
64. Available at: newsroom.varian.com/2016-02-23-Varian-Equipped-MarylandProton-Treatment-Center.

65. Schellhammer SM, Hoffmann AL, Gantz S, Smeets J, van der Kraaij E, Quets S, et al. Integrating a Low-Field Open MR Scanner With a Static Proton Research Beam Line: Proof of Concept. Phys Med Biol (2018) 63:23LT01. doi: 10.1088/1361-6560/aaece8

66. Freeman T. MRI-Guided Proton Therapy: A Status Update. In: Physics World (2019).

67. US 10, 173, 077 B2(45). Date of Patent: Jan. 8, 2019.

68. Inaniwa T, Suzuki M, Sato S, Muramatsu M, Noda A, Iwata Y, et al. Effect of External Magnetic Fields on Biological Effectiveness of Proton Beams. Int J Radiat Oncol Biol Phys :BIOL CONTRIBUTION (2020) 106:597-603. doi: 10.1016/j.ijrobp.2019.10.040

69. Oborn BM, Dowdell S, Metcalf PE, Crozier S, Mohan R, Keall PJ, et al. Proton Beam Deflection in MRI Fields: Implications for MRI-Guided Proton Therapy. Med Phys (2015) 42:2113. doi: 10.1118/1.4916661

70. Available at: newsroom.varian.com/2016-02-23-Varian-Equipped-MarylandProton-Treatment-Ce.

71. Pearson E, de Wilde O, Doyen R, Forton E, Yongen Y, Krier G, et al. Magnet Developments and Commissioning for the IBA Compact Gantry. IEEE
Trans Appl Supercond. (2014) 24 4401004:4 pp. doi: 10.1109/TASC.2013. 2284719

Conflict of Interest: The authors declare that the research was conducted in the absence of any commercial or financial relationships that could be construed as a potential conflict of interest.

Publisher's Note: All claims expressed in this article are solely those of the authors and do not necessarily represent those of their affiliated organizations, or those of the publisher, the editors and the reviewers. Any product that may be evaluated in this article, or claim that may be made by its manufacturer, is not guaranteed or endorsed by the publisher.

Copyright (c) 2022 Collings, Lu, Gupta and Sumption. This is an open-access article distributed under the terms of the Creative Commons Attribution License (CC BY). The use, distribution or reproduction in other forums is permitted, provided the original author(s) and the copyright owner(s) are credited and that the original publication in this journal is cited, in accordance with accepted academic practice. No use, distribution or reproduction is permitted which does not comply with these terms. 


\section{APPENDIX A: MAGNETIC RIGIDITIES OF PROTON- AND CARBON-ION BEAMS}

\section{A1: Magnetic Rigidity of a $250 \mathrm{MeV}$ Proton Beam}

Velocity of light $c=2.998 \times 10^{8} \mathrm{~m} / \mathrm{s}$

Rest mass of proton $m_{o}=1.6712 \times 10^{-27} \mathrm{~kg}$

Charge on proton $q=1.602 \times 10^{-19}$ coulomb (s.A)

Beam kinetic energy $E=250 \mathrm{MeV}=4.006 \times 10^{-11} \mathrm{~J}$

$$
\begin{gathered}
E=m_{o} c^{2}(\gamma-1) \\
\text { hence } \gamma=E /\left(m_{0} c^{2}\right)+1=1.2667 \\
\gamma=1 / \sqrt{ }\left[1-(v / c)^{2}\right] \\
\text { hence } v=c \sqrt{ }\left[1-1 / \gamma^{2}\right]=1.8402 \times 10^{8} \mathrm{~m} / \mathrm{s}
\end{gathered}
$$

Magnetic rigidity, $\boldsymbol{R}=m_{o} w / q=2.432\left(\mathrm{~kg} / \mathrm{s}^{2} . \mathrm{A}\right) \cdot \mathrm{m}$ or T.m

\section{A2: Magnetic Rigidity of a $430 \mathrm{MeV} / \mathrm{u}$ (5.16 GeV) Carbon-Ion Beam}

Rest mass of carbon ion $m_{o}=1.994 \times 10^{-26} \mathrm{~kg}$

Charge on $6+$ carbon ion $q=6 \times 1.602 \times 10^{-19}$ coulomb (s.A)

Beam kinetic energy $E=5.16 \mathrm{GeV}=8.2684 \times 10^{-10} \mathrm{~J}$

$$
\begin{gathered}
E=m_{o} c^{2}(\gamma-1) \\
\text { hence } \gamma=E /\left(m_{o} c^{2}\right)+1=1.461353 \\
\gamma=1 / \sqrt{ }\left[1-(v / c)^{2}\right] \\
\text { hence } v=c \sqrt{ }\left[1-1 / \gamma^{2}\right]=2.18615 \times 10^{8} \mathrm{~m} / \mathrm{s}
\end{gathered}
$$

Magnetic rigidity, $\boldsymbol{R}=m_{o} \gamma / q=6.6275\left(\mathrm{~kg} / \mathrm{s}^{2}\right.$. A). $\mathrm{m}$ or T.m

\section{APPENDIX B: SOME GANTRIES WITH NORMAL-CONDUCTING MAGNETS}

\section{B1: Varian Medical Systems, Inc., Palo Alto, CA, USA}

$\operatorname{Ref}(70)$.

Varian ProBeam ${ }^{\circledR}$ technology is implemented in Gantry 3 of the PROSCAN facility of the Paul Scherrer Institute's (PSI) Center for Proton Therapy (CPT), Villigen, Switzerland (41). The gantry consists of a pair of bending dipoles $\left(45^{\circ}\right.$ and $\left.135^{\circ}\right), 5$ quadrupoles, and 3 orbit correction magnets. A degrader adjusts the proton beam-energy from $70 \mathrm{MeV}$ to $230 \mathrm{MeV}$ and the fields of the magnets downstream change in synchronism. Varian ProBeam ${ }^{\circledR}$ technology is also being implemented at:

$\triangleright$ The Maryland Proton Treatment Center (MPTC), University of Maryland BioPark, West Baltimore, MD, USA

$>$ Scripps Proton Therapy Center, San Diego, CA, USA

$>$ The Rinecker Proton Therapy Center, Munich, Germany
Implementation is also pending at 13 other sites worldwide.

\section{B2: Hitachi, Ltd, Tokyo, Japan}

Ref (42).

Hitachi's system consist of three bending magnets (BM160 upwards, BM2 $60^{\circ}$ downwards and BM3, $90^{\circ}$ downwards) and six quadrupole magnets (5). The gantry length is $6.9 \mathrm{~m}$ and the rotating diameter is $10.0 \mathrm{~m}$. Correction (or steering) magnets compensate for the results of gantry frame distortion during rotation. A beam- energy range of $70 \mathrm{MeV}$ to $250 \mathrm{MeV}$ provides magnetic rigidities, $R$, of 1.23 to $2.43 \mathrm{Tm}$. A maximum magnetic field, $B$, of $1.6 \mathrm{~T}$ calls for a dipole bend radius $\rho=R / B=1.52 \mathrm{~m}$. Hitachi systems (including "PROBEAT-V") have been installed at:

$>$ The Proton Medical Research Center at the University of Tsukuba Hospital, Tsukuba, Japan

$>$ The M.D. Anderson Proton Therapy Center, M.D. Anderson Cancer Center (MDACC), University of Texas, Houston, TX, USA

$>$ The Mayo Clinic Proton Beam Therapy Program, Rochester, MI, USA

$\triangleright$ The Mayo Clinic Proton Beam Therapy Program, Phoenix, AZ, USA

$>$ St. Jude Red Frog Events Proton Therapy Center, Memphis, TN, USA.

\section{B3: Ion Beam Applications (IBA) Louvain- la-Neuve, Belgium}

In IBA's Proteus ${ }^{\circledR} 235$ system a beam of energy $230 \mathrm{MeV}$ extracted from a normal-conducting isochronous cyclotron was adjusted to $60-230 \mathrm{MeV}$ by a graphite degrader and energy selection system. In 2010 IBA claimed that system to be the most commercially successful so far (7). As reported in (7), systems operating in 2010 in the US were located at:

$>$ The Francis H. Burr Proton Therapy Center, Massachusetts General Hospital, Boston

$>$ Midwest Proton Therapy Institute, Bloomington, IN (gantry only)

$\gg$ University of Florida Proton Therapy Institute, Jacksonville, FL

$>$ Roberts Proton Therapy Center, University of Pennsylvania Health System, Philadelphia, PA

$>$ Procure Proton Therapy Center, Oklahoma City, OK

$\triangleright$ Hampton University Proton Therapy Institute, Hampton, VA and elsewhere at:

$>$ Wanjie Proton Therapy Center, Zibo, China

$>$ National Cancer Center, Ilsan, Korea

$\triangleright$ Centre de Protonthérapie de l'Institut Curie, Orsay, France

Many others were under construction or installation. 
The IBA Proteus ${ }^{\circledR} 235$ was succeeded by the IBA ProteusONE ${ }^{\circledR}$ system with its $230 \mathrm{MeV}$ superconducting synchrocyclotron S2C2 (Table 5). Nevertheless beam handling in ProteusONE ${ }^{\circledR}$ was still achieved with normal-conducting magnets. As reported in (71) IBA's compact gantry has a diameter of only $7.2 \mathrm{~m}$. It is furnished with 3 dipole bending magnets, 7 quadrupoles, and 4 steering magnets. The beam energy of $230 \mathrm{MeV}$ dictates a magnetic rigidity $R=2.324 \mathrm{Tm}$ (Table 7); a maximum field, $B$, of $1.41 \mathrm{~T}$ calls for a bending radius $\rho=R / B=1.65 \mathrm{~m}$. ProteusONE ${ }^{\circledR}$ systems have been delivered to:

$\triangleright$ The Rutherford Cancer Centre, South Wales, UK

$\triangleright$ Cyclhad (Cyclotron for Hadron Therapy), Caen, France

$\triangleright$ Hokkaido Ohno Memorial Hospital, Sapporo, Japan 